\title{
Existence of equilibria for economies with externalities and a measure space of consumers ${ }^{\star}$
}

\author{
Bernard Cornet $^{1}$ and Mihaela Topuzu ${ }^{2}$ \\ 1 CERMSEM, Université Paris 1, Pantheon-Sorbonne, 106 bd de l'Hopital, 75013 Paris, FRANCE, \\ and University of Kansas, Lawrence, KS 66045, USA \\ (e-mail: bern@rdcornet.com) \\ 2 CERMSEM, Université Paris 1, Pantheon-Sorbonne, 106 bd de l'Hopital, 75013 Paris, FRANCE, \\ and Universitatea de Vest Timişoara, ROMANIA \\ (e-mail: Mihaela.Topuzu@univ-paris1.fr)
}

Received: May 25, 2004; revised version: October 19, 2004

Summary. This paper considers an exchange economy with a measure space of agents and consumption externalities, which take into account two possible external effects on consumers' preferences: dependence upon prices and dependence upon other agents' consumption. We first consider a model with a general externality mapping and we then treat the particular case of reference coalition externalities, in which the preferences of each agent $a$ are influenced by prices and by the global or the mean consumption of the agents in finitely many (exogenously given) reference coalitions associated with agent $a$. Our paper provides existence results of equilibria in both models when consumers have transitive preferences. It extends in exchange economies the standard results by Aumann [2], Schmeidler [16], Hildenbrand [12], and previous results by Greenberg et al. [11] for price dependent preferences, Schmeidler [17] for fixed reference coalitions and Noguchi [15] for a more particular concept of reference coalitions. We also mention related results obtained independently by Balder [4].

Keywords and Phrases: Externalities, Reference coalitions, Measure space of agents, Equilibrium.

JEL Classification Numbers: D62, D51, H23.

\footnotetext{
* This paper has benefited from comments and valuable discussions with Erik Balder, Stefan Balint, Jean-Marc Bonnisseau, Alessandro Citanna, Gael Giraud, Filipe Martins-da-Rocha, Jean-Philippe Médecin, Jean-François Mertens, Nicholas Yannelis and an anonymous referee.

Correspondence to: $\mathrm{B}$. Cornet
} 


\section{Introduction}

This paper considers an exchange economy with a measure space of agents and consumption externalities, which take into account two possible external effects on consumers' preferences: dependence upon prices and dependence upon other agents' consumptions.

The price dependence externality is a long recognized problem, which recently found new applications in the study of financial markets, where a two-period temporary equilibrium model has a reduced form as a Walrasian model with price dependent preferences. For the existence of equilibria in economies with a measure space of agents and price externalities we refer to Greenberg et al. [11], who use ordered preferences and use a game-theoretical approach, which exploits Debreu's original idea of introducing a price-setting player.

The dependence upon other agents' consumptions has also been considered in recent years, with attempts to have the same level of generality for a measure space of agents as for the case of finitely many agents. In this paper, we will consider agents with transitive strict preferences which are not necessarily complete as in Schmeidler [16]. The question arises if one can drop transitivity and completeness simultaneously but our approach does not cover this case, for which we refer to Khan and Vohra [14] and Yannelis [18]. Our treatment of the existence problem differs from [14] and [18] in considering a weaker convexity assumption on preferences that allows us to encompass the results of Aumann [2], Greenberg et al. [11] and Schmeidler [17]; indeed the way they model externalities does not allow for a convexifying effect on aggregation, even if the measure space is nonatomic.

We first present the model with a general externality mapping. The preference relation of each agent $a$, which may depend upon the externality $e$ in a given externality space $E$, is denoted by $\prec_{a, e}$ and the influence of the externality on agents' preferences is represented by a given (exogenous) externality mapping $\Phi$, which associates to each agent $a$, each price $p$ and each (integrable) consumption allocation $f$, the externality $e=\Phi(a, p, f) \in E$. Thus, given the price $p$ and the allocation $f$ the choices of agent $a$ will be made with the preference relation $\prec_{a, \Phi(a, p, f)}$. Our model considers "finitely many externality effects"; that is, formally, the externality space $E$ is assumed to be a subset of a finite dimensional Euclidean space. This makes an explicit restriction on the couples $(p, f)$ of prices and (integrable) consumption allocation that can influence agents' preferences via the externality mapping $\Phi$.

The previous model allows us to consider the particular case of reference coalitions externalities, in which the preferences of each agent $a$ are influenced by prices and by the global or the mean consumption of the agents in finitely many (exogenously given) reference coalitions associated with agent $a$. Let $(A, \mathcal{A}, \nu)$ be the measure space of consumers, and for each agent $a \in A$ and each price $p$, let $C_{k}(a, p) \in \mathcal{A}(k=1, \ldots, K)$ be finitely many (exogenously given) reference coalitions. Each coalition $C_{k}(a, p) \in \mathcal{A}$ can be considered as the reference class of agent $a$ for a particular group of commodities, say clothes, music, housing or travel. The externality dependence operates via reference consumption vectors (for the particular group of commodities) which can be obtained either as the global 
or as the mean consumption of agents in the reference coalition of agent $a$. With a single reference coalition (i.e., $K=1$ ), the externality mappings $\Phi_{1}$ and $\Phi_{2}$ corresponding to the global and the mean consumption are defined, respectively, by:

$$
\begin{aligned}
& \Phi_{1}(a, p, f)=\int_{C(a, p)} f(\alpha) d \nu(\alpha) ; \\
& \Phi_{2}(a, p, f):= \begin{cases}\frac{1}{\nu[C(a, p)]} \int_{C(a, p)} f(\alpha) d \nu(\alpha) & \text { if } \nu[C(a, p)]>0, \\
0 & \text { if } \nu[C(a, p)]=0 .\end{cases}
\end{aligned}
$$

Both models consider finitely many external effects, with externality space $E=\mathbf{R}_{+}^{H}$, the closed positive orthant of the commodity space $\mathbf{R}^{H}$, denoting by $H$ the number of commodities in the economy. The first case can be illustrated by network effects, i.e., the number of persons connected to a network (internet or mobile phone) and the global consumption in the reference coalition may be important for some agent to decide to connect herself. In the second model, only the mean consumption is used to define the "reference trend".

The aim of this paper is to provide an existence result of equilibria in the model with a general externality mapping and then to deduce from it an existence result in the reference coalitions model both for global and mean dependence. In an exchange economy, we extend the classical results by Aumann [2], Schmeidler [16], Hildenbrand [12], and previous results by Greenberg et al. [11], for price dependent preferences. In the reference coalition model, our result encompasses the result by Schmeidler [17] in the case of constant reference coalitions (i.e., when the coalition does not depend on agent $a$ and the price system). We also generalize the existence result by Noguchi [15] who considers, for each agent $a$, a particular reference coalition, which consists of all the agents who belong to a certain income range associated with agent $a$ (see Sect. 3.3). Finally, we mention the existence results obtained independently by Balder [4], which also generalizes those of $[2,11]$ and [17], without being directly comparable with ours since the externality dependence is defined in a different way and agents have ordered preferences.

The paper is organized as follows. In Section 2, we present the model with a general externality mapping and the associated concept of equilibrium [Sect. 2.1] and we state the main existence results. Our first existence result is stated under a strong convexity assumption on preferences [Sect. 2.2]. We then weaken this convexity assumption [Sect. 2.3] so that we can encompass the Aumann-SchmeidlerHildenbrand existence result in exchange economies. In Section 3, we present the reference coalitions model [Sect. 3.1], and we deduce from our main result the existence of equilibria in this model [Sect. 3.2]. Finally, we present the particular case of a reference coalitions model considered by Noguchi [15] and deduce his existence result. In Section 4 we give the proof of our main existence result [Theorem 2]. We first prove an existence result [Theorem 4] under the additional assumption that the consumption set correspondences are integrably bounded [Sect. 4.1]. We then deduce the main result in the general case from it [Sect. 4.2]. Finally, in the Appendix we present the main properties of the individual quasi-demand correspondence that 
are used in the proof of the existence theorem, along with some properties of the Noguchi's reference coalition and a counterexample due to Balder [4].

\section{The model and the existence result}

\subsection{The model and the equilibrium notion}

We consider an exchange economy with a finite set $H$ of commodities. The commodity space ${ }^{1}$ is represented by the vector space $\mathbf{R}^{H}$.

The set of consumers is defined by a measure space $(A, \mathcal{A}, \nu)$, where $\mathcal{A}$ is a $\sigma$-algebra of subsets in $A$ and $\nu$ is a measure on $\mathcal{A}$. An element $C \in \mathcal{A}$ is a possible group of consumers, also called a coalition. Each consumer $a$ is endowed with a consumption set $X(a) \subset \mathbf{R}^{H}$, an initial endowment $\omega(a) \in \mathbf{R}^{H}$ and a strict preference relation $\prec_{a, e}$ on $X(a)$, which allows for dependence on externalities $e \in E$ (called the externality space), in a way which will be specified hereafter. The set $X(a)$ represents the possible consumptions of consumer $a$. A consumption allocation of the economy specifies the possible consumption of each consumer, and is formally a selection of the correspondence $a \rightarrow X(a)$, which is assumed to be integrable. The set of consumption allocations is denoted by $\mathcal{L}_{X}$. We assume also that the initial endowment mapping $\omega: A \rightarrow \mathbf{R}^{H}$ is integrable and thus the total initial endowment of the economy is $\int_{A} \omega(a) d \nu(a)$.

Specific to this economy is the fact that price externalities and consumption externalities can influence the preference relation of each agent $a$. Thus, given the price $p \in \mathbf{R}^{H}$ and the allocation $f \in \mathcal{L}_{X}$, the choices of agent $a$ will be made with the strict preference relation $\prec_{a, \Phi(a, p, f)}$, where $\Phi: A \times \mathbf{R}^{H} \times \mathcal{L}_{X} \rightarrow E$ is a given mapping, called the externality mapping.

In the presence of externalities, the exchange economy is completely summarized by the couple $(\mathcal{E}, \Phi)$, where the externality space $E$ and the externality mapping $\Phi$ are defined as above and $\mathcal{E}$ specifies the characteristics of the consumers

$$
\mathcal{E}=\left\{\mathbf{R}^{H}, E,(A, \mathcal{A}, \nu),\left(X(a),\left(\prec_{a, e}\right)_{e \in E}, \omega(a)\right)_{a \in A}\right\} .
$$

We now give the definition of an equilibrium in this economy.

Definition 1 An equilibrium of the economy $(\mathcal{E}, \Phi)$ is an element $\left(f^{*}, p^{*}\right) \in \mathcal{L}_{X} \times$ $\mathbf{R}^{H}$ such that $p^{*} \neq 0$ and

(a) [Preference Maximization] for a.e. $a \in A, f^{*}(a)$ is a maximal element for $\prec_{a, e^{*}{ }_{a}}$ in the budget set $B\left(a, p^{*}\right):=\left\{x \in X(a) \mid p^{*} \cdot x \leq p^{*} \cdot \omega(a)\right\}$, where

1 For a finite set $H$ we denote by $\mathbf{R}^{H}$ the set of all mappings from $H$ to $\mathbf{R}$. An element $x$ of $\mathbf{R}^{H}$ will be denoted by $\left(x_{h}\right)_{h \in H}$ or simply by $\left(x_{h}\right)$ when no confusion is possible. For two elements $x=\left(x_{h}\right), x^{\prime}=\left(x_{h}^{\prime}\right)$ in $\mathbf{R}^{H}$, we denote by $x \cdot x^{\prime}=\sum_{h \in H} x_{h} x_{h}^{\prime}$ the scalar product, by $\|x\|=\sqrt{x \cdot x}$ the Euclidean norm and by $B\left(x_{0}, r\right)=\left\{x \in \mathbf{R}^{H} \mid\left\|x-x_{0}\right\| \leq r\right\}$ the closed ball. For $X \subset \mathbf{R}^{H}$, we denote by int $X, \bar{X}$ and $\operatorname{co} X$, respectively, the interior, the closure and the convex hull of $X$. The notations: $x \leq x^{\prime}, x<x^{\prime}, x<<x^{\prime}$ mean, respectively, that for all $h \in H, x_{h} \leq x_{h}^{\prime},\left[x \leq x^{\prime}\right.$ and $\left.x \neq x^{\prime}\right]$, and $x_{h}<x_{h}^{\prime}$; we let $\mathbf{R}_{+}^{H}:=\left\{x \in \mathbf{R}^{H} \mid 0 \leq x\right\}$ and $\mathbf{R}_{++}^{H}:=\left\{x \in \mathbf{R}^{H} \mid 0<<x\right\}$. We also let $\mathbf{1}:=(1, \ldots, 1) \in \mathbf{R}^{H}$ and the canonical basis $\left\{\mathbf{e}^{\mathbf{i}} \mid i \in H\right\}$ of $\mathbf{R}^{H}$ be defined by $e_{h}^{i}=1$, if $h=i$ and $e_{h}^{i}=0$, if $h \neq i$. 
$e^{*}{ }_{a}:=\Phi\left(a, p^{*}, f^{*}\right)$, that is, $f^{*}(a) \in B\left(a, p^{*}\right)$ and there is no $x \in B\left(a, p^{*}\right)$ such that $f^{*}(a) \prec_{a, e_{a}^{*}} x$;

(b) [Market Clearing] $\int_{A} f^{*}(a) d \nu(a)=\int_{A} \omega(a) d \nu(a)$.

\subsection{A first existence result for general externality mappings}

We present the list of assumptions that the economy $(\mathcal{E}, \Phi)$ will be required to satisfy. Let $(A, \mathcal{A}, \nu)$ be a measure space, we recall that a measurable set $\bar{A} \in$ $\mathcal{A}$ is an atom if $\nu(\bar{A})>0$ and for every $C \in \mathcal{A}$ such that $C \subset \bar{A}$, one has $[\nu(C)=0$ or $\nu(\bar{A} \backslash C)=0]$ and we denote by $A_{n a}$ the nonatomic part of $A$; that is, the complementary in $A$ of the union of all the atoms of $A$. We denote by $L^{1}\left(A, \mathbf{R}^{H}\right)$ the space of equivalence classes of integrable mappings from $A$ to $\mathbf{R}^{H}$ and we let $\|f\|_{1}:=\int_{A}\|f(a)\| d \nu(a)$, which defines a norm on $L^{1}\left(A, \mathbf{R}^{H}\right)$. The space $L^{1}\left(A, \mathbf{R}^{H}\right)$ will be endowed with two different topologies, the norm topology defined by the norm $\|f\|_{1}$ and the weak topology $\sigma\left(L^{1}, L^{\infty}\right)$; we recall that a sequence $\left\{f^{n}\right\}$ converges weakly to $f$ if and only if $\sup _{n}\left\|f^{n}\right\|_{1}<\infty$ and $\int_{C} f^{n}(a) d \nu(a) \rightarrow \int_{C} f(a) d \nu(a)$, for every $C \in \mathcal{A}$ (see Dunford and Schwartz [8], p. 291).

Assumption A The measure space $(A, \mathcal{A}, \nu)$ is positive, finite, complete and $L^{1}\left(A, \mathbf{R}^{H}\right)$ is separable for the norm topology;

Assumption C For a.e. $a \in A$, every $(e, x) \in E \times X(a)$ :

(i) $E$ is a closed subset of $\mathbf{R}^{N}$ and $X(a)$ is a closed, convex subset of $\mathbf{R}_{+}^{H}$;

(ii) [Irreflexivity and transitivity] $\prec_{a, e}$ is irreflexive ${ }^{2}$ and transitive ${ }^{3}$;

(iii)[Convexity of preferences on atoms] if a $\in A \backslash A_{n a}$ the set $\left\{x^{\prime} \in X(a) \mid\right.$ not $\left.\left[x^{\prime} \prec_{a, e} x\right]\right\}$ is convex;

(iv)[Continuity] the sets:

$\left\{x^{\prime} \in X(a) \mid x \prec_{a, e} x^{\prime}\right\}$ and $\left\{\left(x^{\prime}, e^{\prime}\right) \in X(a) \times E \mid x^{\prime} \prec_{a, e^{\prime}} x\right\}$

are open, respectively, in $X(a)$ and in $X(a) \times E$ (for their relative topologies);

(v)[Measurability] the consumption set correspondence $a^{\prime} \rightarrow X\left(a^{\prime}\right)$ and the preference correspondence $\left(a^{\prime}, e^{\prime}\right) \rightarrow \prec_{a^{\prime}, e^{\prime}}$ are measurable ${ }^{4}$;

(vi) $\omega \in \mathcal{L}_{X}$, i.e., $\omega: A \rightarrow \mathbf{R}^{H}$ is integrable and $\omega\left(a^{\prime}\right) \in X\left(a^{\prime}\right)$ for a.e. $a^{\prime} \in A$;

Assumption $\mathbf{M}$ (i)[Monotonicity] For a.e. $a \in A, X(a):=\mathbf{R}_{+}^{H}$ and for every $e \in E$ and every $x, x^{\prime}$ in $X(a), x<x^{\prime}$ implies $x \prec_{a, e} x^{\prime}$;

(ii)[Strong survival] $\int_{A} \omega(\alpha) d \nu(\alpha)>>0$.

The above assumptions are standard and need no special comments. In a model without externalities (say $E=\{0\}$ ), they coincide with Aumann-Schmeidler's assumptions, as discussed in the next section.

2 That is, for every $x \in X(a), \operatorname{not}\left[x \prec_{a, e} x\right]$.

3 That is, for every $x, x^{\prime}, x^{\prime \prime} \in X(a), x \prec_{a, e} x^{\prime}$ and $x^{\prime} \prec_{a, e} x^{\prime \prime}$ imply $x \prec_{a, e} x^{\prime \prime}$.

${ }^{4}$ We recall that a correspondence $F$, from a measurable space $(A, \mathcal{A})$ to $\mathbf{R}^{n}$, is said to be $\mathcal{A}$-measurable, or simply measurable, if its graph is a measurable set, i.e., $G_{F}:=\left\{(a, x) \in A \times \mathbf{R}^{H} \mid\right.$ $x \in F(a)\}$ belongs to $\mathcal{A} \otimes \mathcal{B}\left(\mathbf{R}^{n}\right)$, where $\mathcal{B}\left(\mathbf{R}^{n}\right)$ denotes the $\sigma$-algebra of Borel subsets of $\mathbf{R}^{n}$ and $\mathcal{A} \otimes \mathcal{B}\left(\mathbf{R}^{n}\right)$ denotes the $\sigma$-algebra product. The preference correspondence $(a, e) \rightarrow \prec_{a, e}$ is said to be measurable if the correspondence $(a, e) \rightarrow\left\{\left(x, x^{\prime}\right) \in X(a) \times X(a) \mid x \prec_{a, e} x^{\prime}\right\}$ is $\mathcal{A} \otimes \mathcal{B}(E)$-measurable. 
The next assumptions concern the externality side. Hereafter, we suppose that for every $(a, p) \in A \times \mathbf{R}^{H}, \Phi(a, p, f)=\Phi(a, p, g)$ if $f=g$ almost everywhere on $A$. Without any risk of confusion, this allows us to consider $\Phi$ as a mapping $\Phi: A \times \mathbf{R}^{H} \times L_{X} \rightarrow E$, where

$$
L_{X}:=\left\{f \in L^{1}\left(A, \mathbf{R}^{H}\right) \mid f(a) \in X(a) \text { a.e. } a \in A\right\} .
$$

Assumption $\mathbf{E}$ [Caratheodory](i) $E$ is a closed subset of $\mathbf{R}^{N}$;

(ii) for all $(p, f) \in \mathbf{R}_{+}^{H} \times L_{X}$, the mapping $a \rightarrow \Phi(a, p, f)$ is measurable;

(iii) for a.e. $a \in A$, for every sequence $\left\{p^{n}\right\} \subset \mathbf{R}_{+}^{H}$ converging to $p$ and every integrably bounded $d^{5}$ sequence $\left\{f^{n}\right\} \subset L_{X}$ converging weakly to $f$, the sequence $\left\{\Phi\left(a, p^{n}, f^{n}\right)\right\}$ converges to $\Phi(a, p, f)$;

Assumption EB [Boundedness] For all bounded sequence $\left\{\left(p^{n}, f^{n}\right)\right\} \subset \mathbf{R}_{+}^{H} \times L_{X}$ and for a.e. $a \in A$, the sequence $\left\{\Phi\left(a, p^{n}, f^{n}\right)\right\}$ is bounded in $E$.

Assumption $\mathbf{E C}_{0}$ [Convexity of preferences on the nonatomic part] For a.e. $a \in$ $A_{n a}$ and every $(e, x) \in E \times X(a)$, the set $\left\{x^{\prime} \in X(a) \mid \operatorname{not}\left[x^{\prime} \prec_{a, e} x\right]\right\}$ is convex.

The above Caratheodory assumption is a standard regularity assumption. The boundedness assumption $\mathbf{E B}$ will be satisfied, in particular, in the reference coalitions model presented hereafter. We also point out that EB is satisfied when the correspondence $a \rightarrow X(a)$ is integrably bounded (see Assumption IB hereafter) and $\mathbf{C}$ and $\mathbf{E}$ hold.

The last assumption additionally assumes the convexity of preferences on the nonatomic part of $A$ (whereas in $\mathbf{C}$ it was only assumed on the atomic part). This assumption will be discussed and weakened in Section 2.3.

We can now state our first existence result.

Theorem 1 The exchange economy with externalities $(\mathcal{E}, \Phi)$ admits an equilibrium $\left(f^{*}, p^{*}\right)$ with $p^{*}>>0$, if it satisfies Assumptions $\mathbf{A}, \mathbf{C}, \mathbf{M}, \mathbf{E}, \mathbf{E B}$ and $\mathbf{E C}_{0}$.

Theorem 1 is a direct consequence of a more general result [Theorem 2] that will be stated in the following section, which is devoted to the weakening of the convexity assumption $\mathbf{E C}_{0}$.

\subsection{Weakening the convexity assumption $\mathbf{E C}_{0}$}

Since Aumann [2], most of the existence results in models without externalities do not assume convexity of preferences on the nonatomic part $A_{n a}$ of the measure space of consumers (i.e., Assumption $\mathbf{E C}_{0}$ ). To be able to cover Aumann's existence result, we will now weaken the convexity assumption $\mathbf{E C}_{0}$. This will allow us to encompass the known existence results in the three following important cases.

$\mathbf{E}_{1}$ : No externalities [Aumann [2], Schmeidler [16], Hildenbrand [13]] $E_{1}=\{0\}$ and the mapping $\Phi_{1}: A \times \mathbf{R}_{+}^{H} \times L_{X} \rightarrow E_{1}$ is defined by $\Phi_{1}(a, p, f)=0$.

\footnotetext{
5 That is, there is some integrable function $\rho: A \rightarrow \mathbf{R}_{+}$, such that $\sup _{n}\left\|f^{n}(a)\right\| \leq \rho(a)$ for a.e.
} $a \in A$. 
$\mathbf{E}_{2}$ : Price dependent preferences [Greenberg et al. [11]] $E_{2}=\mathbf{R}_{+}^{H}$ and the mapping $\Phi_{2}: A \times \mathbf{R}_{+}^{H} \times L_{X} \rightarrow E_{2}$ is defined by $\Phi_{2}(a, p, f)=p$.

$\mathbf{E}_{3}$ : Constant reference coalitions [Schmeidler [17]] $E_{3}=\left(\mathbf{R}_{+}^{H}\right)^{K}$ and the mapping $\Phi_{3}: A \times \mathbf{R}_{+}^{H} \times L_{X} \rightarrow E_{3}$ is defined by

$$
\Phi_{3}(a, p, f):=\left(\int_{C_{1}} f(a) d \nu(a), \ldots, \int_{C_{K}} f(a) d \nu(a)\right),
$$

where the sets $C_{k}(k=1, \ldots, K)$ are nonempty measurable subsets of $A_{n a}$, which are pairwise disjoint, i.e., $C_{j} \cap C_{k}=\emptyset$ for every $j \neq k$.

In the three above cases, the externality mappings $\Phi_{i}(i=1,2,3)$ are "convex" on $A_{n a}$ in the sense of the following definition (see Proposition 1 below):

Definition 2 We say that the externality mapping $\Phi: A \times \mathbf{R}_{+}^{H} \times L_{X} \rightarrow E$ is "convex" on the measurable set $C \subset A$ if for every $p \in \mathbf{R}_{+}^{H}$, for every $\left\{f_{i}\right\}_{i \in I} \subset$ $L_{X}$ (I finite) and every $f \in L_{X}$ such that

for a.e. $\alpha \in C, f(\alpha) \in \operatorname{co}\left\{f_{i}(\alpha) \mid i \in I\right\}$,

there exists $f^{*} \in L_{X}$ such that:

for a.e. $\alpha \in C, f^{*}(\alpha) \in\left\{f_{i}(\alpha) \mid i \in I\right\}$,

for a.e. $\alpha \in A \backslash C, f^{*}(\alpha)=f(\alpha)$,

for a.e. $a \in A, \Phi(a, p, f)=\Phi\left(a, p, f^{*}\right)$ and $\int_{A} f(\alpha) d \nu(\alpha)=\int_{A} f^{*}(\alpha) d \nu(\alpha)$.

We now can state our main existence result, which extends Theorem 1 and allows us to cover the three above cases $\mathbf{E}_{1}, \mathbf{E}_{2}, \mathbf{E}_{3}$. For this, we need to introduce a new Convexity Assumption EC , which is clearly satisfied in the two important cases: (i) convexity of the preferences on $A_{n a}$ (i.e., Assumption $\mathbf{E C}_{0}$ of Theorem 1), and (ii) "convexity" of $\Phi$ on $A_{n a}$.

Theorem 2 The exchange economy with externalities $(\mathcal{E}, \Phi)$ admits an equilibrium $\left(f^{*}, p^{*}\right)$ with $p^{*}>>0$, if it satisfies Assumptions A, C, M, E, EB, together with the following one:

Assumption EC There exists a measurable set $C \subset A_{n a}$ such that:

(i) for a.e. $a \in A_{n a} \backslash C$, the preferences are convex, that is, for every $(e, x) \in$ $E \times X(a)$, the set $\left\{x^{\prime} \in X(a) \mid \operatorname{not}\left[x^{\prime} \prec_{a, e} x\right]\right\}$ is convex, and

(ii) the externality mapping $\Phi$ is "convex" on $C$.

The proof of Theorem 2 is given in Section 4 and relies on an intermediary result (Theorem 4) in which the monotonicity assumption $\mathbf{M}$ is replaced by the assumption that the consumption correspondence $a \rightarrow X(a)$ is integrably bounded (which is clearly stronger than EB holds). In this case, it is worth pointing out that without Assumption EC, the corresponding existence result (Theorem 4) may not hold as shown in the Appendix with a counterexample due to Balder [4].

We end this section by showing that the three above externality mappings $\Phi_{i}$ $(i=1,2,3)$ satisfy Assumption $\mathbf{E C}$, and also a stronger Assumption $\mathbf{E C}_{1}$ (in which no convexity assumption on preferences is made). 
Proposition 1 (a) In the three above cases $\mathbf{E}_{1}, \mathbf{E}_{2}, \mathbf{E}_{3}$, the externality mappings $\Phi=\Phi_{i}(i=1,2,3)$ satisfy the following assumption:

$\mathbf{E C}_{1}$ There exists a measurable set $C \subset A_{n a}$ such that: (i) the externality mapping $\Phi$ only depends on $f_{\mid C}$, in the sense that, $\Phi(a, p, f)=\Phi(a, p, g)$, if $f_{\mid C}=g_{\mid C}$, and

(ii) the externality mapping $\Phi$ is "convex" on $C$.

(b) If Assumption $\mathbf{E C}_{1}$ holds, then $\Phi$ is "convex" on $A_{n a}$, hence Assumption EC holds.

\section{Proof.}

(a) Assumption $\mathbf{E C}_{1}$ is satisfied for $C=A_{n a}$ for the cases $\mathbf{E}_{1}$ and $\mathbf{E}_{2}$ and for $C=\cup_{k=1}^{K} C_{k}$ for $\mathbf{E}_{3}$. This is a consequence of Lyapunov's theorem, applied to $A_{n a}$ in the first two cases and applied successively to each $C_{k}(k=1, \ldots, K)$ in the latter case.

(b) We show that the externality mapping $\Phi$ is "convex" on $A_{n a}$. Indeed, for every $p \in \mathbf{R}_{+}^{H}$, let $\left\{f_{i}\right\}_{i \in I} \subset L_{X}$ (I finite) and $f \in L_{X}$ such that, for a.e. $\alpha \in A_{n a}$, $f(\alpha) \in \operatorname{co}\left\{f_{i}(\alpha) \mid i \in I\right\}$. Since $\Phi$ is "convex" on $C \subset A_{n a}$ (by $\mathbf{E C}_{1}$ ), there exists an integrable mapping $f^{\prime}: A \rightarrow \mathbf{R}^{H}$ such that, for a.e. $\alpha \in C, f^{\prime}(\alpha) \in\left\{f_{i}(\alpha) \mid i \in\right.$ $I\}$, for a.e. $a \in A, \Phi(a, p, f)=\Phi\left(a, p, f^{\prime}\right)$ and $\int_{C} f(\alpha) d \nu(\alpha)=\int_{C} f^{\prime}(\alpha) d \nu(\alpha)$. From above, for a.e. $\alpha \in A_{n a} \backslash C, f(\alpha) \in \operatorname{co}\left\{f_{i}(\alpha) \mid i \in I\right\}$, hence, from Lyapunov's theorem, there exists an integrable mapping $f^{\prime \prime}: A_{n a} \backslash C \rightarrow \mathbf{R}^{H}$ such that $f^{\prime \prime}(\alpha) \in\left\{f_{i}(\alpha) \mid i \in I\right\}$ and $\int_{A_{n a} \backslash C} f(\alpha) d \nu(\alpha)=\int_{A_{n a} \backslash C} f^{\prime \prime}(\alpha) d \nu(\alpha)$. We consider now the mapping $f^{*}: A \rightarrow \mathbf{R}^{H}$ defined by $f^{*}(\alpha)=f^{\prime}(\alpha)$ for every $\alpha \in C, f^{*}(\alpha)=f^{\prime \prime}(\alpha)$ for every $\alpha \in A_{n a} \backslash C$ and $f^{*}(\alpha)=f(\alpha)$ for every $\alpha \in A \backslash A_{n a}$ and we note that, for a.e. $\alpha \in A_{n a}, f^{*}(\alpha) \in\left\{f_{i}(\alpha) \mid i \in I\right\}$ and for a.e. $\alpha \in A \backslash A_{n a}, f^{*}(\alpha)=f(\alpha)$. Moreover, from above, for a.e. $a \in A$, $\Phi(a, p, f)=\Phi\left(a, p, f^{\prime}\right)=\Phi\left(a, p, f^{*}\right)$ (since $\left.f_{\mid C}^{\prime}=f_{\mid C}^{*}\right)$ and $\int_{A} f(\alpha) d \nu(\alpha)=$ $\int_{A} f^{*}(\alpha) d \nu(\alpha)$.

\section{The reference coalitions model}

\subsection{The model and the existence result}

The general model of an exchange economy with externalities $(\mathcal{E}, \Phi)$ allows us to consider the reference coalitions model that we now present as an extension of Schmeidler's model.

We suppose that, given a price $p \in \mathbf{R}_{+}^{H}$, each agent $a$ has finitely many reference coalitions of agents, $C_{k}(a, p) \in \mathcal{A}(k=1 \ldots K)$, whose consumption choices influence the preferences of agent $a$ in a way defined precisely hereafter. Hence, the reference coalitions may depend upon the agent and also on the price that prevails; this differs from Schmeidler's model, in which the reference coalitions are constant. We will assume that each agent $a$ is influenced either by the global consumption or by the mean consumption of agents in the coalition $C_{k}(a, p)$. 
The "global dependence" case is characterized by the externality space $E:=$ $\left(\mathbf{R}_{+}^{H}\right)^{K}$ and the externality mapping $\Phi_{1}^{\mathcal{C}}: A \times \mathbf{R}_{+}^{H} \times L_{X} \rightarrow E$ defined by

$$
\Phi_{1}^{\mathcal{C}}(a, p, f)=\left(\int_{C_{1}(a, p)} f(\alpha) d \nu(\alpha), \ldots, \int_{C_{K}(a, p)} f(\alpha) d \nu(\alpha)\right) .
$$

The "mean dependence" case, is characterized by the externality space $E:=$ $\left(\mathbf{R}_{+}^{H}\right)^{K}$ and the externality mapping $\Phi_{2}^{\mathcal{C}}: A \times \mathbf{R}_{+}^{H} \times L_{X} \rightarrow E$, defined by

$$
\begin{aligned}
& \Phi_{2}^{\mathcal{C}}(a, p, f)=\left(\Phi_{21}^{\mathcal{C}}(a, p, f), \ldots, \Phi_{2 K}^{\mathcal{C}}(a, p, f)\right), \\
& \Phi_{2 k}^{\mathcal{C}}(a, p, f):= \begin{cases}\frac{1}{\nu\left[C_{k}(a, p)\right]} \int_{C_{k}(a, p)} f(\alpha) d \nu(\alpha) & \text { if } \nu\left[C_{k}(a, p)\right]>0 \\
0 & \text { if } \nu\left[C_{k}(a, p)\right]=0 .\end{cases}
\end{aligned}
$$

The reference coalitions model can thus be summarized by the exchange economies with externalities $\left(\mathcal{E}, \Phi_{1}^{\mathcal{C}}\right)$ and $\left(\mathcal{E}, \Phi_{2}^{\mathcal{C}}\right)$, where

$$
\begin{aligned}
\mathcal{E} & =\left\{\mathbf{R}^{H},\left(\mathbf{R}_{+}^{H}\right)^{K},(A, \mathcal{A}, \nu),\left(X(a),\left(\prec_{a, e}\right)_{\left.\left.e \in\left(\mathbf{R}_{+}^{H}\right)^{K}, \omega(a)\right)_{a \in A}\right\},},\right.\right. \\
\mathcal{C} & :=\left(C_{1}(a, p), \ldots, C_{K}(a, p)\right)_{(a, p) \in A \times \mathbf{R}_{+}^{H}},
\end{aligned}
$$

and the externality mappings $\Phi_{1}^{\mathcal{C}}$ and $\Phi_{2}^{\mathcal{C}}$ are defined as above (and correspond, respectively, to the global and the mean dependence).

Before stating the existence result, we recall the following notations; for $C_{1}$, $C_{2}$ in $\mathcal{A}$, we let $C_{1} \Delta C_{2}:=\left(C_{1} \backslash C_{2}\right) \cup\left(C_{2} \backslash C_{1}\right)$ and we let the characteristic function $\chi_{C_{1}}: A \rightarrow \mathbf{R}$ be defined by $\chi_{C_{1}}(a)=1$ if $a \in C_{1}$ and $\chi_{C_{1}}(a)=0$ if $a \notin C_{1}$.

Theorem 3 The exchange economy with reference coalitions externalities $(\mathcal{E}, \mathcal{C})$ admits an equilibrium $\left(p_{1}^{*}, f_{1}^{*}\right)$ with $p_{1}^{*}>>0$ for global dependence and an equilibrium $\left(p_{2}^{*}, f_{2}^{*}\right)$ with $p_{2}^{*}>>0$ for mean dependence (i.e., $\left(\mathcal{E}, \Phi_{i}^{\mathcal{C}}\right)$ admits an equilibrium $\left.\left(p_{i}^{*}, f_{i}^{*}\right)(i=1,2)\right)$, if it satisfies Assumptions $\mathbf{A}, \mathbf{C}, \mathbf{M}, \mathbf{E} \mathbf{C}_{0}$, together with:

\section{Assumption R [Reference Coalition Side]}

For every $k=1, \ldots, K$ for a.e. $a \in A$ and every $p \in \mathbf{R}_{+}^{H}$ :

(i) $\nu\left[C_{k}(a, p)\right]>0$; (ii) for every $\lambda>0, C_{k}(a, \lambda p)=C_{k}(a, p)$;

(iii) for every sequence $p^{n} \rightarrow p$ in $\mathbf{R}_{+}^{H}, \nu\left[C_{k}\left(a, p^{n}\right) \Delta C_{k}(a, p)\right] \rightarrow 0$;

(iv) the set $\left\{\left(a^{\prime}, a^{\prime \prime}\right) \in A \times A \mid a^{\prime \prime} \in C_{k}\left(a^{\prime}, p\right)\right\} \in \mathcal{A} \otimes \mathcal{A}$.

The proof of Theorem 3 is given in Section 3.2.

\subsection{Proof of Theorem 3}

It is a consequence of Theorem 1 and we only have to prove that the externality mappings $\Phi_{i}^{\mathcal{C}}(i=1,2)$ satisfy Assumptions $\mathbf{E}$ and EB. This will be done in the following three steps, noticing first that $[\mathbf{E}(\mathbf{i})]$ is satisfied. 
Step 1 [E(ii)]: For every $(p, f) \in \mathbf{R}_{+}^{H} \times L_{X}$, the mapping $a \rightarrow \Phi_{i}^{\mathcal{C}}(a, p, f)$ $(i=1,2)$ is measurable on $A$.

Proof. Let $(p, f) \in \mathbf{R}_{+}^{H} \times L_{X}$. We first show that the mapping

$$
a \rightarrow \Phi_{1}^{\mathcal{C}}(a, p, f):=\int_{C(a, p)} f(\alpha) d \nu(\alpha)
$$

is measurable. We notice that the mappings $(a, \alpha) \rightarrow f(\alpha)$ and $(a, \alpha) \rightarrow$ $\chi_{C(a, p)}(\alpha)$ are both measurable on $A \times A$ (endowed with the product $\sigma$-algebra $\mathcal{A} \otimes \mathcal{A})$, from the fact that $f \in L^{1}\left(A, \mathbf{R}_{+}^{H}\right)$ and Assumption $\mathbf{R}(\mathbf{i v})$ respectively. Hence, the mapping $(a, \alpha) \rightarrow \chi_{C(a, p)}(\alpha) f(\alpha)$ is measurable on $A \times A$.

Since $\chi_{C(a, p)}(\alpha) f(\alpha) \leq f(\alpha)$ for a.e. $(a, \alpha) \in A \times A$ and $f \in L^{1}\left(A, \mathbf{R}_{+}^{H}\right)$, applying the measurability part of Fubini's theorem, the mapping

$$
a \rightarrow \int_{A} \chi_{C(a, p)}(\alpha) f(\alpha) d \nu(\alpha)=\int_{C(a, p)} f(\alpha) d \nu(\alpha)
$$

is correctly defined and is measurable on $A$. Hence, the mapping $\Phi_{1}^{\mathcal{C}}$ satisfies Assumption E(ii).

We now show that the mapping

$$
\Phi_{2}^{\mathcal{C}}(a, p, f):= \begin{cases}\frac{1}{\nu[C(a, p)]} \Phi_{1}^{\mathcal{C}}(a, p, f) & \text { if } \nu\left[C_{k}(a, p)\right]>0 \\ 0 & \text { if } \nu\left[C_{k}(a, p)\right]=0\end{cases}
$$

is measurable on $A$. Using the above argument for $f=1$, we deduce that the mapping $a \rightarrow \nu[C(a, p)]$ is measurable on A. Since $\nu[C(a, p)]>0$ for a.e. $a \in A$ (by $\mathbf{R}(\mathbf{i})$ ), in view of the measurability property of $\Phi_{1}^{\mathcal{C}}$, the mapping $\Phi_{2}^{\mathcal{C}}$ satisfies Assumption E(ii).

Step 2 [E(iii)]: For a.e. $a \in A$ and for every sequence $\left\{p^{n}\right\}$ converging to $p$ in $\mathbf{R}_{+}^{H}$ and every integrably bounded sequence $\left\{f^{n}\right\}$ converging weakly to $f$ in $L_{X}$, the sequence $\left\{\Phi_{i}^{\mathcal{C}}\left(a, p^{n}, f^{n}\right)\right\}$ converges to $\Phi_{i}^{\mathcal{C}}(a, p, f)(i=1,2)$.

Proof. Let $\left\{\left(p^{n}, f^{n}\right)\right\}$ as above. We first prove that $\Phi_{1}^{\mathcal{C}}$ satisfies [E(iii)], i.e., for a.e. $a \in A$

$$
\Phi_{1}^{\mathcal{C}}\left(a, p^{n}, f^{n}\right)=\int_{C\left(a, p^{n}\right)} f^{n}(\alpha) d \nu(\alpha) \rightarrow \int_{C(a, p)} f(\alpha) d \nu(\alpha)=\Phi_{1}^{\mathcal{C}}(a, p, f) .
$$

For this, one notices that

$$
\begin{aligned}
& \left\|\int_{C\left(a, p^{n}\right)} f^{n}(\alpha) d \nu(\alpha)-\int_{C(a, p)} f(\alpha) d \nu(\alpha)\right\| \leq \\
& \left\|\int_{C\left(a, p^{n}\right)} f^{n}(\alpha) d \nu(\alpha)-\int_{C(a, p)} f^{n}(\alpha) d \nu(\alpha)\right\|+\left\|\int_{C(a, p)}\left[f^{n}(\alpha)-f(\alpha)\right] d \nu(\alpha)\right\| .
\end{aligned}
$$


For the second term, since $\left\{f^{n}\right\}$ converges weakly to $f$, one has

$$
\left\|\int_{C(a, p)}\left[f^{n}(\alpha)-f(\alpha)\right] d \nu(\alpha)\right\| \rightarrow 0 .
$$

For the first term we have

$$
\begin{aligned}
& \left\|\int_{A} \chi_{C\left(a, p^{n}\right)}(\alpha) f^{n}(\alpha) d \nu(\alpha)-\int_{A} \chi_{C(a, p)}(\alpha) f^{n}(\alpha) d \nu(\alpha)\right\| \leq \\
& \int_{A}\left|\chi_{C\left(a, p^{n}\right)}(\alpha)-\chi_{C(a, p)}(\alpha)\right|\left\|f^{n}(\alpha)\right\| d \nu(\alpha) \leq \\
& \int_{A}\left|\chi_{C\left(a, p^{n}\right)}(\alpha)-\chi_{C(a, p)}(\alpha)\right| \rho(\alpha) d \nu(\alpha)=\int_{C\left(a, p^{n}\right) \Delta C(a, p)} \rho(\alpha) d \nu(\alpha),
\end{aligned}
$$

recalling that the sequence $\left\{f^{n}\right\}$ is integrably bounded, hence, for some integrably function $\rho: A \rightarrow \mathbf{R}_{+}$, one has $\sup _{n}\left\|f^{n}(a)\right\| \leq \rho(a)$ for a.e. $a \in A$. Moreover, for a.e. $a \in A, \nu\left[C\left(a, p^{n}\right) \Delta C(a, p)\right] \rightarrow 0$ when $p^{n} \rightarrow p$ (by $\mathbf{R}($ iii)), hence

$$
\int_{C\left(a, p^{n}\right) \Delta C(a, p)} \rho(\alpha) d \nu(\alpha) \rightarrow 0,
$$

since the mapping $C \rightarrow \int_{C} \rho(\alpha) d \nu(\alpha)$, from $\mathcal{A}$ to $\mathbf{R}_{+}$, is a positive measure, absolutely continuous with respect to $\nu$. This implies that the first term converges to zero ${ }^{6}$ and ends the proof that $\Phi_{1}^{\mathcal{C}}$ satisfies [E(iii)].

We now prove that $\Phi_{2}^{\mathcal{C}}$ satisfies [E(iii)]. Since, for a.e. $a \in A, \Phi_{1}^{\mathcal{C}}\left(a, p^{n}, f^{n}\right) \rightarrow$ $\Phi_{1}^{\mathcal{C}}(a, p, f)$ and $\nu[C(a, p)]>0$ (by $\mathbf{R}(\mathbf{i})$ ), it suffices to show that $\nu\left[C\left(a, p^{n}\right)\right] \rightarrow$ $\nu[C(a, p)]$. Indeed, one has

$$
\begin{aligned}
& \left|\nu\left[C\left(a, p^{n}\right)\right]-\nu[C(a, p)]\right|=\left|\int_{A} \chi_{C\left(a, p^{n}\right)}(\alpha) d \nu(\alpha)-\int_{A} \chi_{C(a, p)}(\alpha) d \nu(\alpha)\right| \\
& \leq \int_{A}\left|\chi_{C\left(a, p^{n}\right)}(\alpha)-\chi_{C(a, p)}(\alpha)\right| d \nu(\alpha)=\nu\left[C\left(a, p^{n}\right) \Delta C(a, p)\right],
\end{aligned}
$$

which converges to zero (by $\mathbf{R}(\mathbf{i i i})$ ) when $p^{n} \rightarrow p$.

Step 3 [EB]: If $\left\{\left(p^{n}, f^{n}\right)\right\} \subset \mathbf{R}_{+}^{H} \times L_{X}$ is a (norm-)bounded sequence, then for a.e. $a \in A$ the sequence $\left\{\Phi_{i}^{\mathcal{C}}\left(a, p^{n}, f^{n}\right)\right\}(i=1,2)$ is bounded in $\left(\mathbf{R}_{+}^{H}\right)^{K}$.

Proof. Let $\left\{\left(p^{n}, f^{n}\right)\right\}$ as above. For a.e. $a \in A$ and for every $n$, one has

$$
0 \leq \Phi_{1}^{\mathcal{C}}\left(a, p^{n}, f^{n}\right)=\int_{C\left(a, p^{n}\right)} f^{n}(\alpha) d \nu(\alpha) \leq \int_{A} f^{n}(\alpha) d \nu(\alpha) .
$$

6 Note: We don't need to use the fact that the sequence $\left\{f^{n}\right\}$ is integrably bounded. Indeed, if $\left\{f^{n}\right\}$ converges weakly to $f$ and $\nu\left[C\left(a, p^{n}\right) \Delta C(a, p)\right] \rightarrow 0$, one has directly

$$
\int_{C\left(a, p^{n}\right) \Delta C(a, p)} f^{n}(\alpha) d \nu(\alpha) \rightarrow 0 .
$$

For details, see Dunford and Schwartz [8] p. 294. Thanks to E. Balder for this remark. 
Since $\left\{f^{n}\right\}$ is norm-bounded and $f^{n} \geq 0$, we deduce that for some $m \geq 0$

$$
\sup _{n}\left\|\Phi_{1}^{\mathcal{C}}\left(a, p^{n}, f^{n}\right)\right\| \leq m .
$$

We now prove that $\Phi_{2}^{\mathcal{C}}\left(a, p^{n}, f^{n}\right)$ is bounded. Indeed, from above, for a.e. $a \in A$ and every $n$, we get

$$
\left\|\Phi_{2}^{\mathcal{C}}\left(a, p^{n}, f^{n}\right)\right\|=\left\|\frac{1}{\nu\left[C\left(a, p^{n}\right)\right]} \Phi_{1}^{\mathcal{C}}\left(a, p^{n}, f^{n}\right)\right\| \leq m \frac{1}{\nu\left[C\left(a, p^{n}\right)\right]},
$$

since $\nu\left[C\left(a, p^{n}\right)\right]>0$ for a.e. $a \in A$. Recalling now that the sequence $\left\{p^{n}\right\}$ is bounded and that in the previous step we have proved that, for a.e. $a \in A$, the mapping $p \rightarrow \frac{1}{\nu[C(a, p)]}$ is continuous on $\mathbf{R}_{+}^{H}$, we get that, for a.e. $a \in A$, there exists $m_{a}^{\prime}>0$ such that $\frac{1}{\nu\left[C\left(a, p^{n}\right)\right]} \leq m_{a}^{\prime}$ for every $n$. It suffices to take for a.e. $a \in A$

$$
m_{a}:=\frac{1}{\min _{\left\{p \in \overline{\left\{p^{n}\right\}} \mid \nu[C(a, p)]>0\right\}} \nu[C(a, p)]} .
$$

Hence, for a.e. $a \in A, \sup _{n}\left\|\Phi_{2}^{\mathcal{C}}\left(a, p^{n}, f^{n}\right)\right\| \leq m_{a}^{\prime} . m$.

\subsection{Noguchi's reference coalitions model}

We now present Noguchi's model (see [15]) and we deduce his existence result from Theorem 3. It can be described by a reference coalition model, with a unique reference coalition $C_{N}(a, p)$, defined, for each consumer $a$ at price system $p$, by

$$
C_{N}(a, p):=\{\alpha \in A \mid p \cdot \omega(\alpha) \in I(\omega(a), \delta(a), p)\},
$$

where $\delta: A \rightarrow \mathbf{R}_{+}^{H}$ is a fixed function and $I(\omega(a), \delta(a), p)$ is a subset of $\mathbf{R}$. Quoting Noguchi [15], "intuitively speaking, $I(\omega(a), \delta(a), p)$ represents (for agent a) an income range in the income-scale, relative to income $p \cdot \omega(a)$ and with magnitude $p \cdot \delta(a)$ " and among the examples given, we point out the following one defined by the interval $I(\omega(a), \delta(a), p)=(p \cdot \omega(a)+p \cdot \delta(a), \infty)$.

We now state the existence result.

Corollary 1 [Noguchi] The economy $\left(\mathcal{E}, \Phi_{2}^{\mathcal{C}_{N}}\right)$ admits an equilibrium, if it satisfies Assumptions $\mathbf{A}, \mathbf{C}, \mathbf{M}, \mathbf{E C}_{0}$ together with:

Assumption $\mathbf{N}$ For every $(a, w, d, p, t) \in A \times\left(\mathbf{R}_{+}^{H}\right)^{3} \times \mathbf{R}_{+}$:

(i) $I(w, d, p)$ is an open subset of $(0, \infty)$;

(ii) $\nu\left[C_{N}(a, p)\right]>0^{7}$; (iii) the function $\delta: A \rightarrow \mathbf{R}_{+}^{H}$ is measurable;

(iv) for every $\lambda>0, I(w, d, \lambda p)=\lambda I(w, d, p)$;

(v) for every sequence $\left\{\left(p_{n}, t_{n}\right)\right\} \subset \mathbf{R}_{+}^{H} \times \mathbf{R},\left(p_{n}, t_{n}\right) \rightarrow(p, t)$, if $t \in I(w, d, p)$, then $t_{n} \in I\left(w, d, p_{n}\right)$ for $n$ large enough;

${ }^{7}$ In fact, Noguchi [15] only assumed that $\nu[C(a, p)]>0$ for every $(a, p) \in A \times \mathbf{R}_{+}^{H}$ such that $p \cdot \omega(a)>0$. To be able to get Noguchi's existence result in the more general case, we need to weaken Assumption $\mathbf{R}$ of Theorem 3 and, also, Assumptions $\mathbf{E}$ and $\mathbf{E B}$ of Theorem 2 as in the working paper [7]. 
(vi) for every sequence $\left\{\left(p_{n}, t_{n}\right)\right\} \subset \mathbf{R}_{+}^{H} \times \mathbf{R},\left(p_{n}, t_{n}\right) \rightarrow(p, t), t_{n} \in I\left(w, d, p_{n}\right)$ implies $t \in \overline{I(w, d, p)}$;

(vii) for every sequence $\left\{\left(w_{n}, d_{n}\right)\right\} \subset \mathbf{R}_{+}^{H} \times \mathbf{R}_{+}^{H},\left(w_{n}, d_{n}\right) \rightarrow(w, d)$, if $t \in$ $I(w, d, p)$, then $t \in I\left(w_{n}, d_{n}, p\right)$ for $n$ large enough;

(viii) for every sequence $\left(w_{n}, d_{n}\right) \rightarrow(w, d)$ in $\mathbf{R}_{+}^{H} \times \mathbf{R}_{+}^{H}, t \in I\left(w_{n}, d_{n}, p\right)$ implies $t \in \overline{I(w, d, p)}$;

(ix) the set $\overline{I(w, d, p)} \backslash I(w, d, p)$ is countable and $c \in \overline{I(\omega(a), \delta(a), p)} \backslash I(\omega(a), \delta(a), p)$ implies $\nu[\{a \in A \mid p \cdot \omega(a)=c\}]=0$.

Proof. We define the reference coalitions $\mathcal{C}:=(C(a, p))_{(a, p) \in A \times \mathrm{R}_{+}^{H}}$ by

$$
C(a, p):=\{\alpha \in A \mid p \cdot \omega(\alpha) \in \overline{I(\omega(a), \delta(a), p)}\} .
$$

From Assumption $\mathbf{N}(\mathbf{i x})$, for every $(a, p) \in A \times \mathbf{R}_{+}^{H}$, we get

$$
C_{N}(a, p) \subset C(a, p) \text { and } \nu\left(C(a, p) \backslash C_{N}(a, p)\right)=0,
$$

hence, $\int_{C_{N}(a, p)} f(\alpha) d \nu(\alpha)=\int_{C(a, p)} f(\alpha) d \nu(\alpha)$ for every $f \in L_{X}$.

Consequently, every equilibrium of $\left(\mathcal{E}, \Phi_{2}^{\mathcal{C}}\right)$ is an equilibrium for $\left(\mathcal{E}, \Phi_{2}^{\mathcal{C}_{N}}\right)$. We now obtain the existence of equilibria of $\left(\mathcal{E}, \Phi_{2}^{\mathcal{C}}\right)$ from Theorem $3(K=1)$ and it suffices to prove that the reference coalitions $\mathcal{C}$, defined above, satisfy Assumption $\mathbf{R}$ of Theorem 3. This is proved in Section 5.2 of the Appendix.

\section{Proof of the existence theorem}

\subsection{Proof of Theorem 2 in the integrably bounded case}

In this section, we provide an intermediary existence result, also of interest for itself, under the following additional assumption:

IB [Integrably Bounded] The correspondence $a \rightarrow X(a)$, from $A$ to $\mathbf{R}_{+}^{H}$, is integrably bounded, that is, for some integrable function $\rho: A \rightarrow \mathbf{R}_{+}$, $\sup _{x \in X(a)}\|x\| \leq \rho(a)$ for a.e. $a \in A$.

Theorem 4 Under Assumptions A, C, E, EC and IB, the economy $(\mathcal{E}, \Phi)$ admits a free-disposal quasi-equilibrium $\left(f^{*}, p^{*}\right) \in \mathcal{L}_{X} \times \mathbf{R}^{H}$ with $p^{*}>0$, in the sense that:

(a) [Preference Maximization] for a.e. $a \in A, f^{*}(a) \in B\left(a, p^{*}\right)$ and for a.e. $a \in A$ such that $p^{*} \cdot \omega(a)>\inf p^{*} \cdot X(a), f^{*}(a)$ is a maximal element for $\prec_{a, e^{*}{ }_{a}}$ in the budget set $B\left(a, p^{*}\right)$ where $e^{*}{ }_{a}:=\Phi\left(a, p^{*}, f^{*}\right)$;

(b) [Market Clearing] $\int_{A} f^{*}(a) d \nu(a) \leq \int_{A} \omega(a) d \nu(a)$.

To prepare the proof of Theorem 4 , we define the "quasi-demand" correspondence $D$, from $A \times \mathbf{R}_{+}^{H} \times E$ to $\mathbf{R}_{+}^{H}$, by

$$
D(a, p, e):= \begin{cases}\left\{x \in B(a, p) \mid \nexists x^{\prime} \in B(a, p), x \prec_{a, e} x^{\prime}\right\} \\ \text { if } \inf p \cdot X(a)<w(a, p) \\ B(a, p) & \text { if } \inf p \cdot X(a)=w(a, p) .\end{cases}
$$


We let $\Delta:=\left\{p \in \mathbf{R}_{+}^{H} \mid \sum_{h} p_{h}=1\right\}$ and we define the correspondence $\Gamma$, from $\Delta \times L_{X}$ to $\Delta \times L_{X}$, by $\Gamma(p, f)=\Gamma_{1}(p, f) \times \Gamma_{2}(p, f)$, where

$$
\begin{aligned}
& \Gamma_{1}(p, f):=\left\{p \in \Delta \mid(p-q) \cdot \int_{A}(f(a)-\omega(a)) d \nu(a) \geq 0 \forall q \in \Delta\right\} \subset \Delta \\
& \Gamma_{2}(p, f):=\left\{g \in L_{X} \mid g(a) \in \operatorname{co} D(a, p, \Phi(a, p, f)) \text { for a.e. } a \in A\right\} \subset L_{X} .
\end{aligned}
$$

The next lemmas summarize the properties of the set $L_{X}$ and of the correspondence $\Gamma$.

Lemma 1 The set $L_{X}$, endowed with the weak topology of the (locally convex) space $L^{1}\left(A, \mathbf{R}^{H}\right)$, is nonempty, convex, compact and metrizable.

Proof. First, the set $L_{X}$ is nonempty, since it contains the mapping $\omega$; indeed $\omega \in L^{1}\left(A, \mathbf{R}^{H}\right)$ and, for a.e. $a \in A, \omega(a) \in X(a)$ (by $\mathbf{C}(\mathbf{v i})$ ). The set $L_{X}$ is also convex, since for a.e. $a \in A, X(a)$ is a convex set (by $\mathbf{C}(\mathbf{i})$ ).

We show now that $L_{X}$ is compact for the weak topology of $L^{1}\left(A, \mathbf{R}^{H}\right)$. From the fact that the correspondence $a \rightarrow X(a)$ is integrably bounded (by IB), one has $\lim _{\nu(C) \rightarrow 0} \int_{C} f(a) d \nu(a)=0$ uniformly for $f \in L_{X}$. Consequently, since $\nu(A)<\infty$, the set $L_{X}$, which is (norm-)bounded, is also weakly sequentially compact (see, for example, Dunford and Schwartz [8], p. 294). In view of EberleinSmulian's Theorem, this is equivalent to the fact that the weak closure of $L_{X}$ is weakly compact. The proof will be complete if we show that $L_{X}$ is weakly closed. But in the normed space $L^{1}\left(A, \mathbf{R}^{H}\right)$, the convex set $L_{X}$ is weakly closed if and only if it is closed in the norm topology of $L^{1}\left(A, \mathbf{R}^{H}\right)$ (see, for example, Dunford and Schwartz [8], p. 422). To show that $L_{X}$ is closed, we consider a sequence $\left\{f^{n}\right\} \subset L_{X}$ which converges to some $f \in L^{1}\left(A, \mathbf{R}^{H}\right)$ for the norm topology of $L^{1}\left(A, \mathbf{R}^{H}\right)$, then there exists a subsequence $\left\{f^{n_{k}}\right\}$, which converges almost everywhere to $f$. But, for a.e. $a \in A, f^{n_{k}}(a) \in X(a)$, since $f^{n_{k}} \in L_{X}$. Taking the limit when $k \rightarrow \infty$, for a.e. $a \in A, f(a) \in X(a)$, since $X(a)$ is a closed set (by C(i)). This ends the proof that $L_{X}$ is weakly compact.

Finally, $L_{X}$ is metrizable (for the weak topology) since, in a separable Banach space, the weak topology on a weakly compact set is metrizable (see, for example, Dunford and Schwartz [8], p. 434).

Lemma 2 The correspondences $\Gamma_{1}$ and $\Gamma_{2}$ defined on $\Delta \times L_{X}$ with values, respectively in $\Delta$ and $L_{X}$, have both a closed graph and non-empty, convex, compact values.

Proof. For the correspondence $\Gamma_{1}$, the proof is a classical argument using Berge's Maximum Theorem (see Berge [5] p. 123) and proving that the function $(p, f) \rightarrow$ $p \cdot\left(\int_{A} f(a) d \nu(a)-\int_{A} \omega(a) d \nu(a)\right)$ is continuous on $\Delta \times L_{X}$. Indeed, this is clearly the case since the scalar product (of $\left.\mathbf{R}^{H}\right)(p, x) \rightarrow p \cdot x$ is continuous and the real-valued functions $(p, f) \rightarrow p$ and $(p, f) \rightarrow \int_{A} f(a) d \nu(a)$ are continuous on $\Delta \times L_{X}$, when $L_{X}$ is endowed with the weak topology of $L^{1}\left(A, \mathbf{R}^{H}\right)$ (recalling that $L_{X}$ is metrizable). 
Consider now the correspondence $\Gamma_{2}$. It has clearly convex values and we show hereafter that it has nonempty values. For every $(p, f) \in \Delta \times L_{X}$

$$
\left\{g \in L_{X} \mid g(a) \in D(a, p, \Phi(a, p, f)) \text {, for a.e. } a \in A\right\} \subset \Gamma_{2}(p, f) .
$$

The existence of a measurable selection of the correspondence

$$
a \rightarrow D(a):=D(a, p, \Phi(a, p, f)) \subset B(0, \rho(a))
$$

is a consequence of Aumann's theorem and it suffices to show that $(i)$ for a.e. $a \in A$, $D(a) \neq \emptyset$ and $(i i)$ the correspondence $D($.$) is measurable. The first assertion is a$ consequence of Proposition 2 of the Appendix. We now prove the second assertion. Indeed,

$$
\begin{aligned}
\mathrm{G}_{D} & =\left\{(a, z) \in A \times \mathbf{R}^{H} \mid z \in D(a)\right\} \\
& =\left\{(a, z) \in A \times \mathbf{R}^{H} \mid(a, \Phi(a, p, f), z) \in G\right\}=h^{-1}(G),
\end{aligned}
$$

where $G:=\left\{(a, e, z) \in A \times E \times \mathbf{R}^{H} \mid z \in D(a, p, e)\right\}$ and $h: A \times \mathbf{R}^{H} \rightarrow$ $A \times E \times \mathbf{R}^{H}$ is defined by $h(a, z)=(a, \Phi(a, p, f), z)$. But the mapping $h$ is clearly measurable, since the mapping $a \rightarrow \Phi(a, p, f)$ is measurable (by $\mathbf{E}(\mathbf{i i})$ ), and $\mathrm{G} \in$ $\mathcal{A} \otimes \mathcal{B}(E) \otimes \mathcal{B}\left(\mathbf{R}^{H}\right)$, since the correspondence $(a, e) \rightarrow D(a, p, e)$ is measurable [Proposition 2 of the Appendix]. Consequently, $\mathrm{G}_{D}=h^{-1}(G) \in \mathcal{A} \otimes \mathcal{B}\left(\mathbf{R}^{H}\right)$, which ends the proof of Assertion $(i i)$.

Finally, every measurable selection of the correspondence $a \rightarrow D(a)$ is integrable, since from Assumption IB, for a.e. $a \in A, D(a) \subset B(0, \rho(a))$ for some integrable function $\rho$. This shows that $\Gamma_{2}(p, f)$ is nonempty.

We now show that the correspondence $\Gamma_{2}$ has a closed graph. Indeed (recalling that $L_{X}$ is metrizable), let $\left\{\left(p^{n}, f^{n}, g^{n}\right)\right\}$ be a sequence converging to some element $(p, f, g)$ in $\Delta \times L_{X} \times L_{X}$ such that $g^{n} \in \Gamma_{2}\left(p^{n}, f^{n}\right) \subset L_{X}$ for all $n$. Since the sequence $\left\{g^{n}\right\}$ is integrably bounded (by $\mathbf{I B}$ ) and converges weakly to $g$ in $L^{1}\left(A, \mathbf{R}^{H}\right)$, it is a standard result (see, for example, Yannelis [19]) that

$$
\text { for a.e. } a \in A, g(a) \in \overline{\mathrm{co}} \operatorname{Ls}\left\{g^{n}(a)\right\} \text {. }
$$

But, for a.e. $a \in A$, the correspondence $(p, f) \rightarrow c o D(a, p, \Phi(a, p, f))$ has a closed graph and convex values, since the correspondence $(p, e) \rightarrow D(a, p, e)$ has a closed graph [Proposition 2 of Appendix] and the mapping $(p, f) \rightarrow \Phi(a, p, f)$ is continuous on $\Delta \times L_{X}$ (by $\mathbf{E}$ (iii), IB and the metrizability of $L_{X}$ ). Hence, recalling that, for a.e. $a \in A, g^{n}(a) \in \operatorname{coD}\left(a, p^{n}, \Phi\left(a, p^{n}, f^{n}\right)\right)$ for all $n$, the closed graph property implies

$$
\operatorname{Ls}\left\{g^{n}(a)\right\} \subset \operatorname{coD}(a, p, \Phi(a, p, f)) .
$$

Consequently, for a.e. $a \in A$

$$
g(a) \in \overline{\operatorname{co}} \operatorname{Ls}\left\{g^{n}(a)\right\} \subset \operatorname{coD}(a, p, \Phi(a, p, f)),
$$

which shows that $g \in \Gamma_{2}(p, f)$ and ends the proof of the lemma.

From the two above lemmas, recalling that the Cartesian product of two correspondences with closed graph and non-empty, convex, compact values is a correspondence with closed graph and non-empty, convex, compact values (see Berge 
[5] p. 121), the space $L:=\mathbf{R}^{H} \times L^{1}\left(A, \mathbf{R}^{H}\right)$, the set $K:=\Delta \times L_{X}$ and the correspondence $\Gamma$ satisfy all the assumptions of the following fixed-point theorem (see, for example, Fan [9] and Glicksberg [10]).

Theorem 5 (Fan-Glicksberg) Let $K$ be a non-empty, convex, compact subset of a Hausdorff locally convex space $L$ and let $\Gamma$ be a correspondence, from $K$ to $K$, with a closed graph and non-empty, convex, compact values. Then there exists $\bar{x} \in K$ such that $\bar{x} \in \Gamma(\bar{x})$.

Consequently, there exists an element $(\bar{p}, \bar{f}) \in \Delta \times L_{X}$ satisfying:

$$
\begin{aligned}
& (\bar{p}-p) \cdot \int_{A}(\bar{f}(a)-\omega(a)) d \nu(a) \geq 0 \text { for all } p \in \Delta, \\
& \bar{f}(a) \in \operatorname{co} D(a, \bar{p}, \Phi(a, \bar{p}, \bar{f})) \text { for a.e. } a \in A
\end{aligned}
$$

The following lemma shows that we can remove the convex hull in the above assertion, by eventually modifying the function $\bar{f}$.

Lemma 3 There exists $f^{*} \in L_{X}$ satisfying:

$$
\begin{aligned}
& (\bar{p}-p) \cdot \int_{A}\left(f^{*}(a)-\omega(a)\right) d \nu(a) \geq 0 \text { for all } p \in \Delta, \\
& f^{*}(a) \in D\left(a, \bar{p}, \Phi\left(a, \bar{p}, f^{*}\right)\right) \text { for a.e. } a \in A .
\end{aligned}
$$

Proof. From Assertion (2) and the fact that the correspondence $a \rightarrow D(a):=$ $D(a, \bar{p}, \Phi(a, \bar{p}, \bar{f}))$, from $A$ to $\mathbf{R}^{H}$, is measurable [Proposition 2 of the Appendix], there exist finitely many measurable selections $f_{i}(i \in I)$ of the correspondence $a \rightarrow D(a)$ such that, for a.e. $a \in A, \bar{f}(a) \in \operatorname{co}\left\{f_{i}(a) \mid i \in I\right\}$. Indeed, consider the correspondence $F$, from $A$ to $\left(\mathbf{R}^{H} \times \mathbf{R}\right)^{\# H+1}$, defined by

$$
\begin{array}{r}
F(a):=\left\{\left(f_{i}, \lambda_{i}\right)_{i=1, \ldots, \# H+1} \mid\left(f_{i}, \lambda_{i}\right) \in D(a) \times \mathbf{R}_{+}, \text {for all } i\right. \\
\left.\sum_{i} \lambda_{i}=1 \text { and } \bar{f}(a)=\sum_{i} \lambda_{i} f_{i}\right\} .
\end{array}
$$

Then, clearly $F$ is measurable and nonempty valued, from Caratheodory's theorem and the fact that $\bar{f}(a) \in \operatorname{co} D(a)$. Consequently, from Aumann's theorem, there exists a measurable selection of the correspondence $F$, which defines the measurable selections $f_{i}$ of the correspondence $a \rightarrow D(a)$.

From Assumption EC, there exists a measurable set $C \subset A_{n a}$ such that:

(i) for a.e. $a \in A_{n a} \backslash C$, the preference relation $\prec_{a, \Phi(a, \bar{p}, \bar{f})}$ is convex and (ii) there exists $f^{*} \in L_{X}$ such that,

$$
\begin{aligned}
& \text { for a.e. } a \in A, \Phi(a, \bar{p}, \bar{f})=\Phi\left(a, \bar{p}, f^{*}\right) \\
& \begin{aligned}
\text { for a.e. } a & \in C, f^{*}(a) \in\left\{f_{i}(a) \mid i \in I\right\} \subset D(a, \bar{p}, \Phi(a, \bar{p}, \bar{f})) \\
& =D\left(a, \bar{p}, \Phi\left(a, \bar{p}, f^{*}\right)\right)
\end{aligned}
\end{aligned}
$$

for a.e. $a \in A \backslash C, f^{*}(a)=\bar{f}(a)$ and $\int_{A} \bar{f}(\alpha) d \nu(\alpha)=\int_{A} f^{*}(\alpha) d \nu(\alpha)$.(6) 
Since the preference relation $\prec_{a, \Phi(a, \bar{p}, \bar{f})}$ is convex for a.e. $a \in A \backslash C$ (first, for

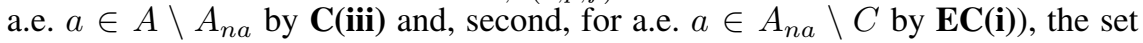
$D(a, \bar{p}, \Phi(a, \bar{p}, \bar{f}))$ is convex. Then, from above

$$
\text { for a.e. } \begin{aligned}
a \in A \backslash C, f^{*}(a) & =\bar{f}(a) \in \operatorname{coD}(a, \bar{p}, \Phi(a, \bar{p}, \bar{f})) \\
& =D\left(a, \bar{p}, \Phi\left(a, \bar{p}, f^{*}\right)\right) .
\end{aligned}
$$

The Assertions (3) and (4) of the lemma follow from Assertions (1),(6) and (5),(7) respectively.

We come back to the proof of Theorem 4 and we show that, for $p^{*}=\bar{p}$, $\left(p^{*}, f^{*}\right)$ is a free disposal quasi-equilibrium of $(\mathcal{E}, \Phi)$. Indeed, from Assertion (4), for a.e. $a \in A, f^{*}(a) \in D\left(a, p^{*}, \Phi\left(a, p^{*}, f^{*}\right)\right)$, hence the equilibrium preference maximization condition is satisfied. This implies, in particular, that for a.e. $a \in A$, $f^{*}(a) \in B\left(a, p^{*}\right)$, hence $p^{*} \cdot f^{*}(a) \leq p^{*} \cdot \omega(a)$. Integrating over $A$, one gets $p^{*} \cdot \int_{A}\left(f^{*}(a)-\omega(a)\right) d \nu(a) \leq 0$. Using Assertion (3), one deduces that

$$
p \cdot \int_{A}\left(f^{*}(a)-\omega(a)\right) d \nu(a) \leq 0 \text { for all } p \in \Delta,
$$

which implies the equilibrium market clearing condition

$$
\int_{A} f^{*}(a) d \nu(a) \leq \int_{A} \omega(a) d \nu(a) .
$$

\subsection{Proof of Theorem 2 in the general case}

\subsubsection{Truncation of the economy}

For each integer $k>1$ and for every $a \in A$, we let

$$
X^{k}(a):=\{x \in X(a) \mid x \leq k[\mathbf{1} \cdot \omega(a)] \mathbf{1}\}
$$

and, for every $e \in E$, we consider the restriction of the preference relation $\prec_{a, e}$ on the set $X^{k}(a)$, which will be denoted identically $\prec_{a, e}$ in the following. We define the truncated economy $\mathcal{E}^{k}$, by

$$
\mathcal{E}^{k}=\left\{\mathbf{R}^{H}, E,(A, \mathcal{A}, \nu),\left(X^{k}(a),\left(\prec_{a, e}\right)_{e \in E}, \omega(a)\right)_{a \in A}\right\},
$$

where the characteristics of $\mathcal{E}^{k}$ are the same as in the economy $\mathcal{E}$, but the consumption sets $X^{k}(a)$ and the preferences $\left(\prec_{a, e}\right)_{e \in E}$ of the agents, which are defined as above.

The externality mapping $\Phi^{k}: A \times \mathbf{R}^{H} \times L_{X}^{k} \rightarrow E$ is defined as the restriction of $\Phi$ to $A \times \mathbf{R}^{H} \times L_{X}^{k}$, where

$$
L_{X}^{k}:=\left\{f \in L^{1}\left(A, \mathbf{R}^{H}\right) \mid f(a) \in X^{k}(a) \text {, for a.e. } a \in A\right\} .
$$

It is easy to see that if $(\mathcal{E}, \Phi)$ satisfies the assumption of Theorem 2 , then for every $k$, $\left(\mathcal{E}^{k}, \Phi^{k}\right)$ satisfies all the assumptions of Theorem 4. Consequently, from Theorem 4 , for every $k$ there exists a free-disposal quasi-equilibrium $\left(p^{k}, f^{k}\right)$ of $\left(\mathcal{E}^{k}, \Phi^{k}\right)$ with $p^{k}>0$. 
4.2.2 For $k$ large enough, $p^{k}>>0$

Lemma 4 There exists $\delta>0$ such that $p^{k} \geq \delta \mathbf{1}$ for $k$ large enough.

Proof. Without any loss of generality, we can assume that the sequence $\left\{p^{k}\right\}$ converges to some element $p^{*}$ in the compact set $\Delta$. To prove the lemma it suffices to show that $p^{*}>>0$.

We first show that, for a.e. $a \in A$

$$
\exists(f(a), e(a)) \in \mathbf{R}_{+}^{H} \times E,\left(p^{*}, f(a), e(a)\right) \in \operatorname{Ls}\left\{\left(p^{k}, f^{k}(a), e^{k}(a)\right)\right\} .
$$

Indeed, since $\left(p^{k}, f^{k}\right)$ is a free-disposal quasi-equilibrium of $\left(\mathcal{E}^{k}, \Phi^{k}\right)$ for every $k$, one has

$$
\text { for a.e. } a \in A, 0 \leq f^{k}(a) \text { and } \int_{A} f^{k}(a) d \nu(a) \leq \int_{A} \omega(a) d \nu(a),
$$

hence the sequence $\left\{\int_{A} f^{k}(a) d \nu(a)\right\}$ is bounded in $\mathbf{R}^{H}$ and we shall deduce that $\sup _{k}\left\|f^{k}\right\|_{1}<\infty$. Defining in $\mathbf{R}^{H},\|x\|_{1}=\sum_{h}\left|x_{h}\right|$ and, recalling that, for some $m>0,\|x\| \leq m\|x\|_{1}$ for every $x$, we get

$$
\begin{aligned}
\left\|f^{k}\right\|_{1}:=\int_{A}\left\|f^{k}(a)\right\| d \nu(a) & \leq m \int_{A} \sum_{h \in H} f_{h}^{k}(a) d \nu(a) \\
& =m\left\|\int_{A} f^{k}(a) d \nu(a)\right\|_{1},
\end{aligned}
$$

since $f^{k}(a) \geq 0$, for a.e. $a \in A$. Consequently, $\sup _{k}\left\|f^{k}\right\|_{1}<\infty$, since the sequence $\left\{\int_{A} f^{k}(a) d \nu(a)\right\}$ is bounded.

Since the sequence $\left\{\left(p^{k}, f^{k}\right)\right\}$ is (norm-)bounded in $\mathbf{R}^{H} \times L^{1}\left(A, \mathbf{R}^{H}\right)$, from Assumption EB, there exists a set $N_{1} \in \mathcal{A}$ with $\nu\left(N_{1}\right)=0$ such that, for all $a \in A \backslash N_{1}$ the sequence $\left\{e^{k}(a)\right\}$ is bounded in $E$, where $e^{k}(a):=\Phi\left(a, p^{k}, f^{k}\right)$.

In view of the standard version of Fatou's lemma, one has

$$
\int_{A} \liminf \left\|f^{k}(a)\right\| d \nu(a) \leq \liminf \int_{A}\left\|f^{k}(a)\right\| d \nu(a)=\liminf \left\|f^{k}\right\|_{1}<\infty,
$$

since from above $\sup _{n}\left\|f^{k}\right\|_{1}<\infty$. Consequently, there exists $N_{2} \in \mathcal{A}$ with $\nu\left(N_{2}\right)=0$ such that, for all $a \in A \backslash N_{2}$, liminf $\left\|f^{k}(a)\right\|<\infty$, which implies that, for all $a \in A \backslash N_{2}$, there exists a subsequence $\left\{k_{n}(a)\right\}$ such that the sequence $\left\{f^{k_{n}(a)}(a)\right\}$ is bounded in $\mathbf{R}_{+}^{H}$.

Let $a \in A \backslash\left(N_{1} \cup N_{2}\right)$, noticing that the sequence $\left\{\left(f^{k_{n}(a)}(a), e^{k_{n}(a)}(a)\right)\right\}$ is bounded in $\mathbf{R}_{+}^{H} \times E$, without any loss of generality we can assume that the sequence $\left\{\left(f^{k_{n}(a)}(a), e^{k_{n}(a)}(a)\right)\right\}$ converges to some element $(f(a), e(a)) \in \mathbf{R}_{+}^{H} \times E$. Hence, Assertion (8) holds for all $a \in A \backslash\left[N_{1} \cup N_{2}\right]$.

We now come back to the proof of Lemma 4. We choose a particular agent $a_{0} \in A$ for whom the following properties hold: $(i)$ the preferences of agent $a_{0}$ are continuous, $(i i)$ the preferences of agent $a_{0}$ are monotonic; $(i i i)$ $p^{*} \cdot \omega\left(a_{0}\right)>0$ and there exists a subsequence $\left\{k_{n}\right\}$, depending on $a_{0}$, such that 
$(i v)\left(p^{k_{n}}, f^{k_{n}}\left(a_{0}\right), e^{k_{n}}\left(a_{0}\right)\right) \rightarrow\left(p^{*}, f\left(a_{0}\right), e\left(a_{0}\right)\right)$, for some $\left(f\left(a_{0}\right), e\left(a_{0}\right)\right) \in$ $\mathbf{R}_{+}^{H} \times E,(v)$ for every $n, f^{k_{n}}\left(a_{0}\right) \in D^{k_{n}}\left(a_{0}, p^{k_{n}}, e^{k_{n}}\left(a_{0}\right)\right)$ with $e^{k_{n}}\left(a_{0}\right)=$ $\Phi\left(a_{0}, p^{k_{n}}, f^{k_{n}}\right)$. Such an agent $a_{0}$ clearly exists, since each of the above Assertions $(i)-(v)$ hold for a.e. $a \in A$; they correspond, respectively, to Assumption C(iv), $\mathbf{M}(\mathbf{i}), \mathbf{M}(\mathbf{i i})$, Assertion (8) and the equilibrium preference maximization condition for $\left(p^{k_{n}}, f^{k_{n}}\right)$ for every $n$.

We will now show that $p^{*}>>0$. Suppose it is not true, then there exists $h$ such that $p_{h}^{*}=0$. From the above properties of agent $a_{0}$, for all $n, p^{k_{n}} \cdot f^{k_{n}}\left(a_{0}\right) \leq$ $p^{k_{n}} \cdot \omega\left(a_{0}\right), p^{k_{n}} \rightarrow p^{*}$ and $f^{k_{n}}\left(a_{0}\right) \rightarrow f\left(a_{0}\right)$, and at the limit one gets $p^{*} \cdot f\left(a_{0}\right) \leq$ $p^{*} \cdot \omega\left(a_{0}\right)$. Since agent $a_{0}$ has monotonic preferences, there exists $z=f\left(a_{0}\right)+t \mathbf{e}^{\mathbf{h}}$, for some $t>0$ such that $f\left(a_{0}\right) \prec_{a_{0}, e\left(a_{0}\right)} z$ and clearly $p^{*} \cdot z=p^{*} \cdot f\left(a_{0}\right) \leq$ $p^{*} \cdot \omega\left(a_{0}\right)$. We now show that

$$
\exists z^{\prime} \in \mathbf{R}_{+}^{H}, p^{*} \cdot z^{\prime}<p^{*} \cdot \omega\left(a_{0}\right), f\left(a_{0}\right) \prec_{a_{0}, e\left(a_{0}\right)} z^{\prime} .
$$

Indeed, if $p^{*} \cdot z<p^{*} \cdot \omega\left(a_{0}\right)$, we take $z^{\prime}=z$. If $p^{*} \cdot z=p^{*} \cdot \omega\left(a_{0}\right)>0$, we can choose $i \in H$ such that $p_{i}^{*}>0$ and $z_{i}>0$. Since agent $a_{0}$ has continuous preferences, there exists $\varepsilon>0$ such that $z^{\prime}=z-\varepsilon \mathbf{e}^{\mathbf{i}} \in \mathbf{R}_{+}^{H}$ and $f\left(a_{0}\right) \prec_{a_{0}, e\left(a_{0}\right)} z^{\prime}$. We have also $p^{*} \cdot z^{\prime}=p^{*} \cdot z-\varepsilon p_{i}^{*}<p^{*} \cdot \omega\left(a_{0}\right)$. This ends the proof of Assertion (9).

We end the proof by contradicting the fact that $f^{k_{n}}\left(a_{0}\right)$ belongs to $D^{k}\left(a_{0}, p^{k_{n}}, e^{k_{n}}\left(a_{0}\right)\right)$. Indeed, from $p^{*} \cdot \omega\left(a_{0}\right)>0$ (by $\left.(i i i)\right)$ and Assertion (9), recalling that the sequence $\left\{\left(p^{k_{n}}, f^{k_{n}}\left(a_{0}\right), e^{k_{n}}\left(a_{0}\right)\right)\right\}$ converges to $\left(p^{*}, f\left(a_{0}\right), e\left(a_{0}\right)\right)$ (by $(i v)$ ) and using the continuity of preferences of agent $a_{0}$, for $n$ large enough, we get $p^{k_{n}} \cdot \omega\left(a_{0}\right)>0, z^{\prime} \in \mathbf{R}_{+}^{H}, p^{k_{n}} \cdot z^{\prime} \leq p^{k_{n}} \cdot \omega\left(a_{0}\right)$ and $x^{k_{n}}\left(a_{0}\right) \prec_{a_{0}, e^{k_{n}}\left(a_{0}\right)} z^{\prime}$. Moreover, we can also assume that $z^{\prime} \in X^{k_{n}}\left(a_{0}\right)$. All together, these conditions contradict the fact that $f^{k_{n}}\left(a_{0}\right) \in D^{k}\left(a_{0}, p^{k_{n}}, e^{k_{n}}\left(a_{0}\right)\right)$ and this ends the proof of the lemma.

\subsubsection{For $k$ large enough, $\left(p^{k}, f^{k}\right)$ is an equilibrium for $(\mathcal{E}, \Phi)$}

It is a consequence of the following lemma.

Lemma 5 For every $k$ large enough and for a.e. $a \in A$, one has:

(i) $B\left(a, p^{k}\right) \subset X^{k}(a)$;

(ii) $f^{k}(a)$ is a maximal element in $B\left(a, p^{k}\right)$ for $\prec_{a, e^{k}(a)}$, where $e^{k}(a)=\Phi\left(a, p^{k}, f^{k}\right)$;

(iii) $p^{k} \cdot f^{k}(a)=p^{k} \cdot \omega(a)$;

(iv) $\int_{A} f^{k}(a) d \nu(a)=\int_{A} \omega(a) d \nu(a)$.

Proof. From Lemma 4, there exists $K$ such that, for every $k \geq K$

$$
p_{h}^{k}>\delta \text { for each } h \in H \text { and } \frac{1}{\delta} \leq k .
$$

In the following we fix $k \geq K$.

(i) For a.e. $a \in A$, let $x \in B\left(a, p^{k}\right)$, i.e., $x \in \mathbf{R}_{+}^{H}$ and $p^{k} \cdot x \leq p^{k} \cdot \omega(a)$. From above, recalling that $p^{k} \in \Delta$, one gets

$$
\delta x_{h} \leq p_{h}^{k} x_{h} \leq p^{k} \cdot x \leq p^{k} \cdot \omega(a) \leq \sum_{h} \omega_{h}(a)=\mathbf{1} \cdot \omega(a),
$$


which implies that

$$
0 \leq x \leq \frac{1}{\delta}[\mathbf{1} \cdot \omega(a)] \mathbf{1} \leq k[\mathbf{1} \cdot \omega(a)] \mathbf{1}
$$

or equivalently $x \in X^{k}(a)$.

(ii) For a.e. $a \in A$ such that $p^{k} \cdot \omega(a)>0, f^{k}(a)$ is a maximal element in $B\left(a, p^{k}\right)$ for $\prec_{a, e^{k}(a)}$, since $B\left(a, p^{k}\right) \subset X^{k}(a)$ (by Part $\left.(i)\right)$ and the fact that $\left(p^{k}, f^{k}\right)$ is a free-disposal quasi-equilibrium for $\left(\mathcal{E}^{k}, \Phi^{k}\right)$. For a.e. $a \in A$ such that $p^{k} \cdot \omega(a)=0$, recalling that $p^{k}>>0$ (by Lemma 4 ), we get $B\left(a, p^{k}\right)=\{0\}$ and the result follows from the Irreflexivity Assumption C(ii).

(iii) The result is obvious for a.e. $a \in A$ such that $p^{k} \cdot \omega(a)=0$. Assume now that $p^{k} \cdot \omega(a)>0$. From the Monotonicity Assumption $\mathbf{M}(\mathbf{i})$, there exists a sequence $\left\{f^{n}(a)\right\} \subset \mathbf{R}_{+}^{H}$ such that $f^{n}(a) \rightarrow f^{k}(a)$ and $f^{k}(a) \prec_{a, e^{k}(a)} f^{n}(a)$. From Part $(i i), f^{k}(a)$ is a maximal element of $\prec_{a, e^{k}(a)}$ in $B\left(a, p^{k}\right)$, consequently $p^{k} \cdot f^{n}(a)>$ $p^{k} \cdot \omega(a)$. Passing to the limit one gets $p^{k} \cdot f^{k}(a) \geq p^{k} \cdot \omega(a)$, which together with $f^{k}(a) \in B\left(a, p^{k}\right)$ implies that $p^{k} \cdot f^{k}(a)=p^{k} \cdot \omega(a)$.

(iv) Integrating over $A$ the equalities of Part (iii), one gets

$$
p^{k} \cdot\left(\int_{A} f^{k}(a) d \nu(a)-\int_{A} \omega(a) d \nu(a)\right)=0 .
$$

Since $\left(p^{k}, f^{k}\right)$ is a free-disposal quasi-equilibrium for $\left(\mathcal{E}^{k}, \Phi^{k}\right)$, one has

$$
\int_{A} f^{k}(a) d \nu(a) \leq \int_{A} \omega(a) d \nu(a)
$$

and, recalling that $p^{k}>>0$ (by Lemma 4), we get

$$
\int_{A} f^{k}(a) d \nu(a)=\int_{A} \omega(a) d \nu(a) .
$$

\section{Appendix}

\subsection{Properties of the quasi-demand correspondence}

Let $(A, \mathcal{A}, \nu)$ be a measure space of consumers, and assume that each consumer $a$ is endowed with a consumption set $X(a) \subset \mathbf{R}^{H}$, a preference relation $\prec_{a, e}$ on $X(a)$ (for each externality $e \in E$ ) and a wealth mapping $w: A \times \mathbf{R}^{H} \rightarrow \mathbf{R}$. In the following, we let

$$
\begin{aligned}
P & :=\left\{p \in \mathbf{R}^{H} \mid \inf p \cdot X(a) \leq w(a, p) \text { for a.e. } a \in A\right\}, \\
B(a, p) & :=\{x \in X(a) \mid p \cdot x \leq w(a, p)\}, \\
D(a, p, e) & := \begin{cases}\left\{x \in B(a, p) \mid \nexists x^{\prime} \in B(a, p), x \prec_{a, e} x^{\prime}\right\} \\
B(a, p) & \text { if } \inf p \cdot X(a)<w(a, p) \\
\text { if } \inf p \cdot X(a)=w(a, p) .\end{cases}
\end{aligned}
$$

The properties of the quasi-demand correspondence $D$ are summarized in the following proposition, which extends standard results (see, for example, Hildenbrand [12]) in the no-externality case (say $E=\{0\}$ ). 
Proposition 2 Let $\left\{(A, \mathcal{A}, \nu), E,\left(X(a),\left(\prec_{a, e}\right)_{e \in E},\right)_{a \in A}, w\right\}$ satisfy Assumptions $\mathbf{A}, \mathbf{C}$ and $\mathbf{I B}$ and assume that the wealth distribution $w: A \times \mathbf{R}^{H} \rightarrow \mathbf{R}$ is a Caratheodory function ${ }^{8}$. Then:

(i) for every $p \in P$ the correspondence $(a, e) \rightarrow D(a, p, e)$, from $A \times E$ to $\mathbf{R}^{H}$, is measurable;

(ii) for a.e. $a \in A$ the correspondence $(p, e) \rightarrow D(a, p, e)$, from $P \times E$ to $\mathbf{R}^{H}$, has a closed graph and nonempty, compact values.

Proof. In the following, for a.e. $a \in A$ and for every $p \in P$, we let

$$
\begin{aligned}
& P_{a}:=\{p \in P \mid \inf p \cdot X(a)<w(a, p)\}, \\
& A_{p}:=\{a \in A \mid \inf p \cdot X(a)<w(a, p)\} .
\end{aligned}
$$

Proof of $(i)$. Let $p \in P$, we prove that

$$
G:=\left\{(a, e, d) \in A \times E \times \mathbf{R}^{H} \mid d \in D(a, p, e)\right\} \in \mathcal{A} \otimes B(E) \otimes \mathcal{B}\left(\mathbf{R}^{H}\right)
$$

and we first notice that $G=G_{1} \cup G_{2}$, where

$$
\begin{gathered}
\mathrm{G}_{1}:=\left\{(a, e, d) \in\left(A \backslash A_{p}\right) \times E \times \mathbf{R}^{H} \mid d \in X(a), p \cdot d \leq w(a, p)\right\}, \\
\mathrm{G}_{2}:=\left\{(a, e, d) \in A_{p} \times E \times \mathbf{R}^{H} \mid d \in D(a, p, e)\right\} .
\end{gathered}
$$

We notice that $\mathrm{G}_{1} \in \mathcal{A} \otimes B(E) \otimes \mathcal{B}\left(\mathbf{R}^{H}\right)$, since the mapping $(a, d) \rightarrow p \cdot d-w(a, p)$ and the correspondence $a \rightarrow X(a)$ are measurable and $A_{p} \in \mathcal{A}$.

To show that $\mathrm{G}_{2} \in \mathcal{A} \otimes \mathcal{B}(E) \otimes \mathcal{B}\left(\mathbf{R}^{H}\right)$, we apply the argument used by Hildenbrand [12]. Since the correspondence $B(., p)$, from $A_{p}$ to $\mathbf{R}^{H}$, has nonempty values and is measurable, there exists a sequence of measurable mappings $\left\{f_{n}\right\}$, from $A_{p}$ to $\mathbf{R}^{H}$, such that for a.e. $a \in A_{p},\left\{f_{n}(a)\right\}$ is dense in $B(a, p)$ (see, for example, [6]). We now define the correspondences $\xi_{n}$, from $A_{p} \times E$ to $\mathbf{R}^{H}$, by

$$
\xi_{n}(a, e)=\left\{x \in B(a, p) \mid \operatorname{not}\left[x \prec_{a, e} f_{n}(a)\right]\right\}
$$

and we claim that: $D(a, p, e)=\cap_{n=1}^{\infty} \xi_{n}(a, e)$, for a.e. $a \in A_{p}$.

Clearly, for every $n, D(a, p, e) \subset \xi_{n}(a, e)$. Conversely, let $x \in \cap_{n=1}^{\infty} \xi_{n}(a, e)$ and suppose that $x \notin D(a, p, e)$. Then, the set $U=\left\{x^{\prime} \in B(a, p) \mid x \prec_{a, e} x^{\prime}\right\}$ is nonempty and is open relative to $B(a, p)$ (by $\mathbf{C}(\mathbf{i v})$ ). Since the sequence $\left\{f_{n}(a)\right\}$ is dense in $B(a, p)$, we deduce that for some $n_{0}, x \prec_{a, e} f_{n_{0}}(a)$, but this contradicts the fact that $x \in \xi_{n_{0}}(a, e)$. Thus, we have

$$
\begin{aligned}
\mathrm{G}_{2} & =\left\{(a, e, d) \in A_{p} \times E \times \mathbf{R}^{H} \mid d \in D(a, p, e)\right\} \\
& =\cap_{n=1}^{\infty}\left\{(a, e, d) \in A_{p} \times E \times \mathbf{R}^{H} \mid d \in \xi_{n}(a, e)\right\} .
\end{aligned}
$$

Hence, the set $\mathrm{G}_{2}$ is measurable, since $\prec_{a, e}$ is measurable (by $\mathbf{C}(\mathbf{v})$ ), the mappings $f_{n}$ and the correspondence $a \rightarrow B(a, p)$ are measurable and recalling that $A_{p} \in \mathcal{A}$.

${ }^{8}$ That is, for every $p \in \mathbf{R}^{H}$, the function $a \rightarrow w(a, p)$ is measurable and, for a.e. $a \in A$, the function $p \rightarrow w(a, p)$ is continuous. We note that the wealth distribution considered in our model $w(a, p):=p \cdot \omega(a)$ satisfies this property, when $\omega$ is assume to be measurable. 
Proof of $($ ii $)$. We first show that $D(a, p, e) \neq \emptyset$ for a.e. $a \in A$ and every $(p, e) \in$ $P \times E$. For a.e. $a \in A \backslash A_{p}, D(a, p, e)=B(a, p) \neq \emptyset$ since inf $p \cdot X(a) \leq w(a, p)$. We now consider $a \in A_{p}$ and we simply denote $B:=B(a, p)$, which is clearly a nonempty, compact set (by IB). We suppose, by contraposition, that $D(a, p, e)=\emptyset$, that is, for every $x \in B$, there exists $x^{\prime} \in B, x \prec_{a, e} x^{\prime}$. Then $B=\cup_{x^{\prime} \in B} V_{x^{\prime}}$, where $V_{x^{\prime}}=\left\{x \in B \mid x \prec_{a, e} x^{\prime}\right\}$ is open in $B$ (by $\mathbf{C}(\mathbf{i v})$ ). Since $B$ is compact, there exists a finite subset $\left\{x_{i}^{\prime} \mid i \in N\right\} \subset B$ such that $B=\cup_{i \in N} V_{x_{i}^{\prime}}$. We now claim that there exists $i \in N$ such that not $\left[x_{i}^{\prime} \prec_{a, e} x_{j}^{\prime}\right]$ for every $j \in N$. Indeed, if such a maximal element does not exist, for every $i \in N$, there exists $\sigma(i) \in N$ such that $x_{i}^{\prime} \prec_{a, e} x_{\sigma(i)}^{\prime}$. The mappings $\sigma: N \rightarrow N$ clearly admits a cycle, that is, for some $i$ and some integer $k$ one has $i=\sigma^{k}(i)$ (the composition of $\sigma$ with itself $k$ times). The transitivity (by C(ii)) of $\prec_{a, e}$ implies that $x_{i}^{\prime} \prec_{a, e} x_{\sigma^{k}(i)}^{\prime}=x_{i}^{\prime}$ which contradicts the irreflexivity (by $\mathbf{C}$ (ii)) of $\prec_{a, e}$. We end the proof by considering such a maximal element $x_{i}^{\prime} \in B$, which belongs to some set $V_{x_{j}^{\prime}}(j \in N)$, that is, $x_{i}^{\prime} \prec_{a, e} x_{j}^{\prime}$ for some $j \in J$. But this is in contradiction with the maximality of $x_{i}^{\prime}$. This ends the proof that $D(a, p, e)$ is nonempty.

We now show that, for a.e. $a \in A$, the correspondence $(p, e) \rightarrow D(a, p, e)$, from $P \times E$ to $\mathbf{R}^{H}$, has a closed graph. Let $\left(p^{n}, e^{n}, x^{n}\right) \rightarrow(p, e, x)$ in $P \times E \times \mathbf{R}^{H}$ such that, for all $n, x^{n} \in D\left(a, p^{n}, e^{n}\right)$. From $p^{n} \cdot x^{n} \leq w\left(a, p^{n}\right)$, passing to the limit and recalling that the mapping $w(a,$.$) is continuous, one gets p \cdot x \leq w(a, p)$. Recalling that $X(a)$ is closed, we get that $x \in B(a, p)$. Thus, if inf $p \cdot X(a)=w(a, p)$, we have $x \in D(a, p, e)=B(a, p)$. We assume now that inf $p \cdot X(a)<w(a, p)$. Since $p^{n} \rightarrow p$, for $n$ large enough, $w\left(a, p^{n}\right)>\inf p^{n} \cdot X(a)$. Suppose now that $x \notin D(a, p, e)$. This implies that there exists $x^{\prime} \in B(a, p)$ such that $x \prec_{a, e} x^{\prime}$. From the fact that $w(a, p)>\inf p \cdot X(a)$ and the Continuity Assumption C(iv), we can find $x^{\prime \prime} \in X(a)$ such that $x \prec_{a, e} x^{\prime \prime}$ and $p \cdot x^{\prime \prime}<w(a, p)$. Since $p^{n} \rightarrow p$, for $n$ large enough, $p^{n} \cdot x^{\prime \prime}<w\left(a, p^{n}\right)$. Since $e^{n} \rightarrow e$, from the Continuity Assumption C(iv), for $n$ large enough, $x^{n} \prec_{a, e^{n}} x^{\prime \prime}$. Consequently, we can choose $n$ (large enough) such that $w\left(a, p^{n}\right)>\inf p^{n} \cdot X(a), x^{\prime \prime} \in B\left(a, p^{n}\right)$ and $x^{n} \prec_{a, e^{n}} x^{\prime \prime}$, but this contradicts the fact that $x^{n} \in D\left(a, p^{n}, e^{n}\right)$.

\subsection{Properties of Noguchi's reference coalitions}

In this section, we end the proof of Corollary 1 (of Sect. 3.3) and it only remains to show that the reference coalitions, defined by

$$
C(a, p)=\{\alpha \in A \mid p \cdot \omega(\alpha) \in \overline{I(\omega(a), \delta(a), p)}\}
$$

satisfy Assumption $\mathbf{R}$ of Theorem 3.

Proof. $\mathbf{R}(\mathbf{i})$ is a consequence of $\mathbf{N}(\mathbf{i i})$ since $C_{N}(a, p) \subset C(a, p)$ and $\mathbf{R}($ ii) is a direct consequence of $\mathbf{N}(\mathbf{i v})$.

Proof of $\mathbf{R}$ (iii). Let $(a, p) \in A \times \mathbf{R}_{+}^{H}$, we define

$$
W(a, p):=\left\{\omega^{\prime} \in \mathbf{R}_{+}^{H} \mid p \cdot \omega^{\prime} \in I(\omega(a), \delta(a), p)\right\} .
$$


Clearly, one has

$$
\begin{aligned}
& \overline{W(a, p)} \backslash W(a, p) \subset\left\{\omega^{\prime} \in \mathbf{R}_{+}^{H} \mid p \cdot \omega^{\prime} \in \overline{I(\omega(a), \delta(a), p)} \backslash I(\omega(a), \delta(a), p)\right\} \\
& \omega^{-1}(\overline{W(a, p)} \backslash W(a, p)) \subset \cup_{c \in \overline{I(\omega(a), \delta(a), p)} \backslash I(\omega(a), \delta(a), p)}\{\alpha \in A \mid p \cdot \omega(\alpha)=c\}
\end{aligned}
$$

and using Assumption N(ix), one gets

$$
\nu\left[\omega^{-1}(\overline{W(a, p)} \backslash W(a, p))\right]=0 .
$$

Since the measure $\tau:=\nu o \omega^{-1}$ is a finite Borel measure on $\mathbf{R}_{+}^{H}$, from Noguchi [15] (see Lemma 2), for every sequence $\left\{p_{n}\right\} \subset \mathbf{R}_{+}^{H}$ converging to $p$, one has

$$
\tau\left(W\left(a, p_{n}\right) \Delta W(a, p)\right):=\nu\left[\omega^{-1}\left(W\left(a, p_{n}\right) \Delta W(a, p)\right)\right] \rightarrow 0 .
$$

Noticing that $C_{N}\left(a, p^{n}\right)=\omega^{-1}\left(W\left(a, p_{n}\right)\right)$ and $C_{N}(a, p)=\omega^{-1}(W(a, p))$, one gets

$$
\begin{aligned}
\nu\left[C_{N}\left(a, p^{n}\right) \Delta C_{N}(a, p)\right] & =\nu\left[\omega^{-1}\left(W\left(a, p_{n}\right)\right) \Delta \omega^{-1}(W(a, p))\right] \\
& =\nu\left[\omega^{-1}\left(W\left(a, p_{n}\right) \Delta W(a, p)\right)\right] \rightarrow 0 .
\end{aligned}
$$

Recalling now that $\nu\left[C(a, p) / C_{N}(a, p)\right]=0$ for every $(a, p) \in A \times \mathbf{R}_{+}^{H}$, from above, we get $\nu\left[C\left(a, p^{n}\right) \Delta C(a, p)\right] \rightarrow 0$ when $p_{n} \rightarrow p$ in $\mathbf{R}_{+}^{H}$.

Proof of $\mathbf{R}(\mathbf{i v})$. It is a consequence of the following lemma, defining, for a fixed $p \in \mathbf{R}_{+}^{H}$, the mappings $f: A \rightarrow\left(\mathbf{R}_{+}^{H}\right)^{2}, g: A \rightarrow \mathbf{R}_{+}^{H}$ and the correspondence $F$, from $\left(\mathbf{R}_{+}^{H}\right)^{2}$ to $\mathbf{R}$, by $f(a)=(\omega(a), \delta(a)), g(\alpha)=p \cdot \omega(\alpha)$ and $F(\omega, \delta)=$ $I(\omega, \delta, p)$ and noticing that

$$
C(a, p)=\{\alpha \in A \mid g(\alpha) \in \overline{F(f(a))}\}
$$

and that, Condition $\mathbf{N}$ implies that $f, g$ and $F$ satisfy the assumption of the lemma. (We only notice that, $\mathbf{N}(\mathbf{v i i})$ implies that for every $t \in \mathbf{R}_{+}^{H}$, the set $F^{-1}(t):=$ $\left\{(\omega, \delta) \in\left(\mathbf{R}_{+}^{H}\right)^{2} \mid t \in I(\omega, \delta, p)\right\}$ is open, hence measurable.)

Lemma 6 Let $f: A \rightarrow \mathbf{R}^{m}, g: A \rightarrow \mathbf{R}^{n}$ be two measurable mappings and let $F$ be a correspondence, from $\mathbf{R}^{m}$ to $\mathbf{R}^{n}$, such that, for every $(x, t) \in \times \mathbf{R}^{m} \times \mathbf{R}^{n}$, $F(x)$ is open and $F^{-1}(t)$ is measurable. Then the set

$$
G:=\{(a, \alpha) \in A \times A \mid g(\alpha) \in \overline{F(f(a))}\}
$$

is measurable.

Proof. Note that $(a, \alpha) \in G$ if and only if

$$
\forall k \in \mathbb{N}, B\left(g(\alpha), \frac{1}{k}\right) \cap F(f(a)) \neq \emptyset
$$

and, using the fact that $F(f(a))$ is an open set, if and only if

$$
\forall k \in \mathbb{N}, \exists t_{k} \in \mathbb{Q}^{n},\left\|t_{k}-g(\alpha)\right\|<\frac{1}{k} \text { and } t_{k} \in F(f(a)) .
$$


Consequently

$$
G=\cap_{k} \cup_{t \in \mathbb{Q}^{n}}\left[A \times\left\{\alpha \in A \mid\|t-g(\alpha)\|<\frac{1}{k}\right\} \cap\{a \in A \mid t \in F(f(a))\} \times A\right],
$$

which is measurable since the set $\left\{\alpha \in A \mid\|t-g(\alpha)\|<\frac{1}{k}\right\}$ is measurable (since the mapping $g$ is measurable) and the set $\{a \in A \mid t \in F(f(a))\}$ is measurable (since the set $F^{-1}(t)$ is measurable and the mapping $f$ is measurable).

\subsection{Balder's counterexample of nonexistence of equilibria}

Theorem 4 may not hold if we remove the Convexity Assumption EC.

We consider the following example, due to Balder [4], of an economy $\mathcal{E}$ with a single commodity, $A=[0,1]$ endowed with the Lebesgue measure $\nu$ and the Lebesgue $\sigma$-algebra (i.e. the completion of the Borel $\sigma$-algebra). For each agent $a \in[0,1]$, the consumption set and the initial endowment are given by $X(a)=$ $[0,2]$ and $\omega(a)=2$; the preference relation $\prec_{a, e}$ is defined by the utility function $u_{a, e}(x):=|x+e|$. The simplex of prices for this economy is $\Delta=\{1\}$, the externality space is $R$ and the externality mapping $\Phi: A \times \Delta \times L_{X} \rightarrow \mathbf{R}$ is defined by

$$
\Phi(a, 1, f):=a-1-\int_{0}^{a} f(\alpha) d \nu(\alpha) .
$$

It is easy to check that this economy satisfies all the assumptions of Theorem 4, but the convexity assumption EC (i.e., it satisfies Assumptions A, C, E and IB).

From Balder [4], this economy does not admit a free disposal quasi-equilibrium. For the sake of completeness the argument goes as follows. Assume that $\mathcal{E}$ admits such an equilibrium, denoted $\left(f^{*}, 1\right)$. For a.e. $a \in A$, we have $B(a, 1)=[0,2]$, and from the equilibrium consumer condition we deduce that $f^{*}(a)=0$ if $\int_{0}^{a} f^{*}(\alpha) d \nu(\alpha)>a$ and $f^{*}(a)=2$ if $\int_{0}^{a} f^{*}(\alpha) d \nu(\alpha)<a$. We now consider the absolutely continuous function $\Psi: A \rightarrow \mathbf{R}_{+}$defined by $\Psi(a):=$ $\left[\int_{0}^{a}\left(f^{*}(\alpha)-1\right) d \nu(\alpha)\right]^{2}$. From above, we deduce that

$$
\Psi^{\prime}(a)=2\left(f^{*}(a)-1\right) \int_{0}^{a}\left(f^{*}(\alpha)-1\right) d \nu(\alpha) \leq 0 \text { for a.e. } a \in A .
$$

Hence, for every $a \in A \Psi(a) \leq \Psi(0)=0$, which together with $0 \leq \Psi(a)$, implies that $\int_{0}^{a}\left(f^{*}(\alpha)-1\right) d \nu(\alpha)=0$. Consequently, $f^{*}=1$, which contradicts the above assertion that $f^{*}(a) \in\{0,2\}$ for a.e. $a \in A$.

Finally, we show that the Convexity Assumption EC does not hold. Indeed, assume that EC holds. We first notice that for every $a \in A$, for $e=-1$ (which is for example obtained by $\Phi$ with $f=1$ ) and for $x=\frac{1}{2}$, the set

$$
\left\{x^{\prime} \in[0,2] \mid \operatorname{not}\left[x^{\prime} \prec_{a, e} x\right]\right\}=\left[0, \frac{1}{2}\right] \cup\left[\frac{3}{2}, 2\right],
$$

is not convex. Consequently, by Assumption EC, the externality mapping $\Phi$ must be convex on $A$ (in the sense of Definition 2). So, let $\left\{f_{i}\right\}_{i=1,2}$ defined by $f_{1}=0$ 
and $f_{2}=2$, then the function $f=1$ satisfies $f(\alpha) \in \operatorname{co}\left\{f_{1}(\alpha), f_{2}(\alpha)\right\}$ for a.e. $\alpha \in A$. Since $\Phi$ is convex on $A$, there exists $f^{*} \in L_{X}$ such that, for a.e. $\alpha \in A$, $f^{*}(\alpha) \in\left\{f_{1}(\alpha), f_{2}(\alpha)\right\}$ and $\Phi\left(a, 1, f^{*}\right)=\Phi(a, 1, f)$. From this last relation one gets that $\int_{0}^{a} f^{*}(\alpha) d \nu(\alpha)=\int_{0}^{a} f(\alpha) d \nu(\alpha)$ for a.e. $a \in A$, which implies that $f^{*}(\alpha)-f(\alpha)=0$ for a.e. $\alpha \in A$. So, $f^{*}=f=1$, which contradicts the above assertion that $f^{*}(\alpha) \in\{0,2\}$ for a.e. $\alpha \in A$.

\section{References}

1. Arrow, K.J., Debreu, G: Existence of an equilibrium for a competitive economy. Econometrica 22, 265-290 (1954)

2. Aumann, R.J.: Existence of a competitive equilibrium in markets with a continuum of traders. Econometrica 34, 1-17 (1966)

3. Aumann, R.J.: Measurable utility and measurable choice theorem. La Décision, Centre National de la Recherche Scientifique Paris, pp. 15-26 (1967)

4. Balder, E.J.: Existence of competitive equilibria in economies with a measure space of consumers and consumption externalities. Working paper (2003)

5. Berge, C.: Espaces topologiques, functions multivoques. Paris: Dunod 1959

6. Castaing, C., Valadier, M.: Convex analysis and measurable multifunctions. In: Dold, A., Eckmann, B.(eds.) Lecture notes in mathematics, 580. Berlin Heidelberg New York: Springer 1977

7. Cornet, B., Topuzu, M.: Equilibria and externalities. Cahiers de la MSE Université Paris 1 (2003)

8. Dunford, N., Schwartz, J.: Linear operators. New York: Interscience 1966

9. Fan, K.: Fixed-point and min-max theorems in locally convex linear spaces. Proceedings of the National Academy of Sciences USA 39, 121-126 (1952)

10. Glicksberg, I.L.: Generalization of Kakutani fixed-point theorem with applications to Nash equilibrium points. Proceedings of the American Mathematical Society 3, 170-174 (1952)

11. Greenberg, J., Shitovitz, B., Wieczorek, A.: Existence of equilibria in atomless production economies with price-dependent preferences. Journal of Mathematical Economics 6, 31-41 (1979)

12. Hildenbrand, W.: Existence of equilibria for economies with production and a measure space of consumers. Econometrica 38, 608-623 (1970)

13. Hildenbrand, W.: Core and equilibrium of a large economy. Princeton: Princeton University Press 1974

14. Khan, M.A., Vohra, M.: Equilibrium in abstract economies without ordered preferences and with a measure space of agents. Journal of Mathematical Economics 13, 133-142 (1984)

15. Noguchi, M.: Interdependent preferences with a continuum of agents. Journal of Mathematical Economics (forthcoming)

16. Schmeidler, D.: Competitive equilibria in markets with a continuum of traders and incomplete preferences. Econometrica 37, 578-585 (1969)

17. Schmeidler, D.: Equilibrium points of nonatomic games. Journal of Statistical Physics 7, 295-300 (1973)

18. Yannelis, N.C.: Equilibria in noncooperative models of competition. Journal of Economic Theory 41, 96-111 (1987)

19. Yannelis, N.C.: Weak sequential convergence in $L_{p}(\mu, X)$. Journal of Mathematical Analysis and Applications 141, 72-83 (1989) 